NISTIR 6881

\title{
Thermal Performance of Fire Fighters' Protective Clothing. 1. Numerical Study of Transient Heat and Water Vapor Transfer
}

Kuldeep Prasad

William Twilley

J. Randall Lawson

National Institute of Standards and Technology • Technology Administration • U.S. Department of Commerce 


\title{
Thermal Performance of Fire Fighters' Protective Clothing. 1. Numerical Study of Transient Heat and Water Vapor Transfer
}

\author{
Kuldeep Prasad \\ William Twilley \\ J. Randall Lawson \\ Fire Research Division \\ Building and Fire Research Laboratory \\ National Institute of Standards and Technology \\ Gaithersburg, MD 20899-8660
}

August 2002

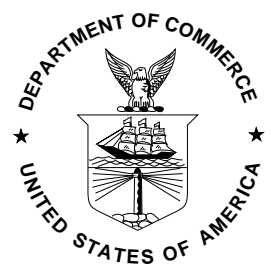

U.S. DEPARTMENT OF COMMERCE

Donald L. Evans, Secretary

TECHNOLOGY ADMINISTRATION

Phillip J. Bond, Under Secretary of Commerce for Technology

NATIONAL INSTITUTE OF STANDARDS AND TECHNOLOGY

Arden L. Bement, Jr., Director
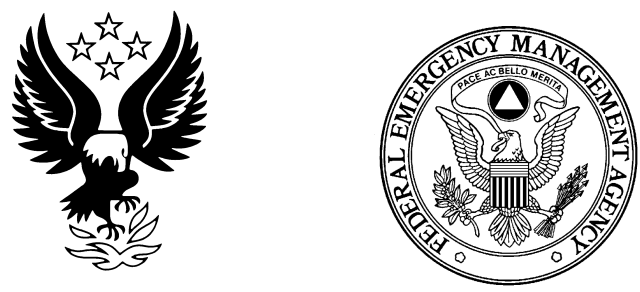

Sponsored in part by:

FEDERAL EMERGENCY MANAGEMENT ADMINISTRATION

Joe M. Allbaugh, Director

U.S. FIRE ADMINISTRATION

R. David Paulison, Administrator 


\section{Contents}

1 Abstract 1

2 Introduction $\quad 1$

3 Detailed Model of Transient Heat and Moisture Transfer Through Cloth 3

4 Approximate Model of Transient Heat and Moisture Transfer Through Cloth 6

5 Thermal Radiation Model $\quad 8$

6 Numerical Model $\quad 11$

6.1 Initial Conditions, Boundary Condition and Interface Conditions . . . . . . . . . . . 11

7 Results and Discussion $\quad 12$

7.1 Heat and Mass Transfer through a Wet Thermal Liner . . . . . . . . . . . . . . . . 12

7.2 Time Dependent Simulations . . . . . . . . . . . . . . . . . . . 17

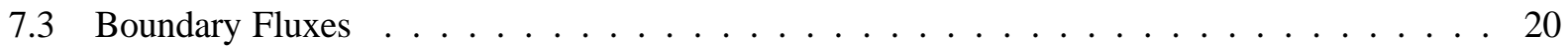

7.4 Wet Turnout Coat Simulation . . . . . . . . . . . . . . . . 22

8 Concluding Remarks $\quad 25$

9 Directions for Future Research Activities 2

10 Acknowledgments $\quad 28$

$\begin{array}{lr}\text { References } & 29\end{array}$ 


\section{List of Figures}

1 Schematic cross-section of a three layered firefighter protective clothing ensemble surrounded by ambient air. Radiative fluxes incident on material boundaries have been indicated. . . . . 9

2 Grid spacing and dimensions for a one-dimensional control volume used in the model for fire fighter protective clothing. . . . . . . . . . . . . . . . . . . . 10

3 Schematic of an Aralite thermal liner consisting of Kevlar batting quilted to an Aramid face cloth used for studying heat and moisture transfer. The batting and face cloth are $0.265 \mathrm{~cm}$ and $0.025 \mathrm{~cm}$ thick, respectively and are separted by a $0.075 \mathrm{~cm}$ thick air gap. . . . . . . . .

4 Comparison of numerical and experimental results of temperature history at the front and back faces of the Arlaite thermal liner. The batting is subjected to an incident flux of 2.5 $\mathrm{kW} / \mathrm{m}^{2}$ for $750 \mathrm{~s}$, followed by a cool down period of $100 \mathrm{~s} . \ldots \ldots \ldots$

5 Comparison of numerically predicted temperature histories for the dry and wet thermal liners. For each case, numerical results have been shown for the front face and the back face. . 15

6 Temperature time histories at the various fabric - air interfaces. The total weight of water $(\mathrm{g})$ in the fabric is plotted as a function of time for the Aralite thermal liner subjected to an incident radiative flux. . . . . . . . . . . . . . . . . .

7 Simulation results at time $t=0 \mathrm{~s}$ for a wet thermal liner subjected to an external incident flux $2.5 \mathrm{~kW} / \mathrm{m}^{2}$. Temperature (top left sub-figure), vapor pressure (top right sub-figure), moisture content (bottom left sub-figure) and dry / wet density (bottom right sub-figure) has been shown as a function of distance measured from the front face of the thermal liner. . . . 18

8 Simulation results at time $t=100 \mathrm{~s}$ for a wet thermal liner subjected to an external incident flux $2.5 \mathrm{~kW} / \mathrm{m}^{2}$. Temperature (top left sub-figure), vapor pressure (top right sub-figure), moisture content (bottom left sub-figure) and dry / wet density (bottom right sub-figure) has been shown as a function of distance measured from the front face of the thermal liner. . . .

9 Simulation results at time $t=200 \mathrm{~s}$ for a wet thermal liner subjected to an external incident flux $2.5 \mathrm{~kW} / \mathrm{m}^{2}$. Temperature (top left sub-figure), vapor pressure (top right sub-figure), moisture content (bottom left sub-figure) and dry / wet density (bottom right sub-figure) has been shown as a function of distance measured from the front face of the thermal liner. . . .

10 Simulation results at time $t=300 \mathrm{~s}$ for a wet thermal liner subjected to an external incident flux $2.5 \mathrm{~kW} / \mathrm{m}^{2}$. Temperature (top left sub-figure), vapor pressure (top right sub-figure), moisture content (bottom left sub-figure) and dry / wet density (bottom right sub-figure) has been shown as a function of distance measured from the front face of the thermal liner. . . .

11 Simulation results at time $t=400 \mathrm{~s}$ for a wet thermal liner subjected to an external incident flux $2.5 \mathrm{~kW} / \mathrm{m}^{2}$. Temperature (top left sub-figure), vapor pressure (top right sub-figure), moisture content (bottom left sub-figure) and dry / wet density (bottom right sub-figure) has been shown as a function of distance measured from the front face of the thermal liner. . . .

12 Simulation results at time $t=500 \mathrm{~s}$ for a wet thermal liner subjected to an external incident flux $2.5 \mathrm{~kW} / \mathrm{m}^{2}$. Temperature (top left sub-figure), vapor pressure (top right sub-figure), moisture content (bottom left sub-figure) and dry / wet density (bottom right sub-figure) has been shown as a function of distance measured from the front face of the thermal liner. . . .

13 Simulation results at time $t=800 \mathrm{~s}$ for a wet thermal liner subjected to an external incident flux $2.5 \mathrm{~kW} / \mathrm{m}^{2}$. Temperature (top left sub-figure), vapor pressure (top right sub-figure), moisture content (bottom left sub-figure) and dry / wet density (bottom right sub-figure) has been shown as a function of distance measured from the front face of the thermal liner. . . . 24

14 Numerical predictions of convective heat flux at the boundaries (front and back faces) as a

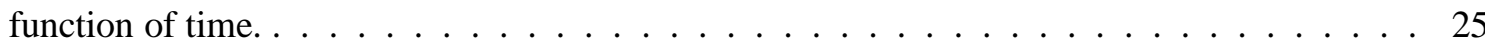


15 Numerical predictions of radiative heat flux at the boundaries (front and back faces) as a function of time. . . . . . . . . . . . . . . . . . . 26

16 Numerical predictions of moisture mass flux at the boundaries (front and back faces) as a function of time. . . . . . . . . . . . . . . . . . . . . 27

17 Schematic of a typical three layered turnout coat used as firefighter protective clothing, subjected to an incident radiative flux. . . . . . . . . . . . . . . . . . . 28

18 Comparison of numerically predicted temperature histories for the dry and wet turnout coat. For each case, numerical results have been shown for the front and back faces. The mass loss curve during evaporation of water from the turnout coat has also been shown on the $Y_{2}$

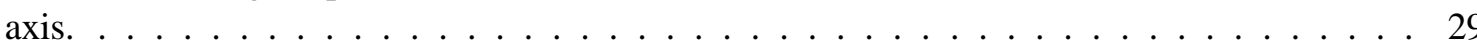

19 Simulation results at time $t=100 \mathrm{~s}$ for a wet turnout coat subjected to an external incident flux of $2.5 \mathrm{~kW} / \mathrm{m}^{2}$. Temperature (top left sub-figure), vapor pressure (top right sub-figure), moisture content (bottom left sub-figure) and dry / wet density (bottom right sub-figure) has been shown as a function of distance measured from the front face of the turnout coat. . . . . 30

20 Simulation results at time $t=600 \mathrm{~s}$ for a wet turnout coat subjected to an external incident flux of $2.5 \mathrm{~kW} / \mathrm{m}^{2}$. Temperature (top left sub-figure), vapor pressure (top right sub-figure), moisture content (bottom left sub-figure) and dry / wet density (bottom right sub-figure) has been shown as a function of distance measured from the front face of the turnout coat. . . . . 31 


\section{Abstract}

This study is the first in a series of reports on understanding the performance of fire fighter clothing, in protecting skin from thermal injury that results from exposure to high intensity thermal radiation. A detailed mathematical model is constructed to study transient heat and moisture transfer through multi-layered fabric assemblies with or without air gaps. The model accounts for changes in thermodynamic and transport properties of the fabric due to the presence of moisture. Numerical simulations are performed to study heat and mass transport through wet thermal liners (used in fire fighter protective clothing) when subjected to a radiative heat flux from a gas fired radiant panel. Results were found to compare well with experimental measurements. The numerical solutions are further analyzed to provide a detailed physical understanding of the governing processes. Moisture in the cloth tends to vaporise upon heating and part of it recondenses in the interior of the cloth. It was observed that the temperature of the fabric layers and total heat flux to the skin is significantly influenced by the amount of moisture and the distribution of moisture in the protective clothing. Finally, simulations are performed for a wet turnout coat assembly to demonstrate the flexibility of the model for designing fire fighter protective clothing.

\section{Introduction}

During fire fighting, fire fighters are subjected to a variety of fire conditions. Fire fighters can be burned by radiant energy that is produced by a fire and localized flame contact exposure. The most common exposure is to low level radiant heat flux over prolonged periods of time. Fire fighters working in these conditions, often sweat profusely, which leads to accumulation of moisture in the turnout garment. Fire fighters can also become wet when they are doused by water from a hose spray. Fire fighters have received second degree burns while working in these "wet" conditions. The presence of moisture in protective clothing can significantly change the garment's protective performance [1]. Wet garments exhibit higher heat transfer rates than dry garments. Evaporation and condensation of the moisture and the energy associated with phase change can affect the temperature and energy flux to the skin surface. Burn injuries can result from heating and evaporation of moisture trapped within one's protective clothing. These injuries are generally referred to as scald injury or steam burns. A numerical tool for studying moisture transport through a thermally irradiated fire fighters' protective clothing is critical in preventing burn injury and in designing new protective gear. Such a tool can also provide information regarding the heat capacity of the protective garment to fire fighters and will assist them in understanding the thermal response of the protective gear to different fire scenarios.

The thermal performance of fire fighters' protective clothing has been a point of interest and discussion for several decades. Many of these discussions are based on fire services field experience. A great deal still needs to be done in terms of detailed scientific analysis for predicting the thermal performance of protective clothing throughout the range of fire environments normally faced by a fire fighter. At a fundamental level, this problem can be viewed as the study of heat and mass (moisture) transfer through porous textile fabrics. Combined heat and mass transfer with phase change in a porous medium is a process which occurs frequently in nature as well as in various engineering endeavors. Drying and humidification in chemical processes and in climate control or movement of moisture in the soil are examples. Such processes have therefore been widely studied by agricultural, civil, chemical and petroleum engineers.

Heat and mass transfer in wet porous media are coupled in a complicated way. The structure of the solid matrix varies widely in shape. There is, in general, a distribution of void sizes, and the structures may also be locally irregular. Energy transport in such a medium occurs by conduction in all of the phases as well as by convection within the liquid and gas phases. Mass transport occurs within the voids of the medium. In an unsaturated state these voids are partially filled with a liquid, whereas the rest of the voids contain some gas. 
For the analysis of wet fire fighter fabrics, the liquid is water and the gas is air. Evaporation or condensation occurs at the interface between the water and air so that the air is mixed with water vapor. A flow of the mixture of air and vapor may be caused by external forces, for instance, by an imposed pressure difference. The vapor will also move relative to the gas by diffusion from regions where the partial pressure of the vapor is higher to those where it is lower. The partial pressure of vapor at the interface to the liquid is determined by the sorption isotherm, which makes it dependent on moisture content as well as on temperature. The saturation pressure is also different on a curved surface from that on a plane one and is influenced by the presence of air. The flow of liquid is caused by external forces, like imposed pressure differences, gravity and internal forces like capillary, intermolecular and osmotic forces.

A detailed study of the transport processes occurring within the solid matrix and in the voids is complicated even for a regularly shaped matrix and is impossible for the irregular shaped configurations that exist in general, in porous media. The normal approach in an analysis is, therefore, to treat the media involved as continua. The energy and mass flux then have to be described by constitutive equations. The various driving forces involved make the constitutive equations quite complicated. The difficulty in handling them is aggravated by the fact that the transport properties involved vary strongly with the structure of the porous medium, the moisture content, and the temperature.

Simultaneous differential equations for the transfer of heat and moisture in porous media under the combined influence of gravity and gradients of temperature and moisture content were developed by D. A. De Vries [2]. These equations are a generalization of those derived by Philip et al. [3]. Eckert and Faghri [4] have performed a general analysis of moisture migration in a slab of an unsaturated porous material for a condition where the temperature of one surface is suddenly increased to a higher value whereas the temperature of the other surface is maintained constant. Udell [5], [6] has derived a general, one-dimensional, steady-state model describing the heat and mass transfer within a homogeneous porous medium, saturated with a wetting liquid, its vapor and a non-condensible gas. The effects of gas diffusion, phase change, conduction, liquid and vapor transport, capillarity, and gravity are included. The analysis is based on a general thermodynamic description of the unique equilibrium states characteristic of liquid wetting porous media. Bouddour et al. [7] have provided a systematic, rigorous and unified treatment of the governing equations for simultaneous heat and mass transfer within a wide range of porous media.

Some work has also been done in the area of coupled diffusion of moisture and heat in hygroscopic textile materials. Gibson [8] has given a review of numerical modeling of convection, diffusion and phase changes in textiles. The paper summarizes current and past work aimed at utilizing CFD techniques for clothing applications. It was shown that water in a hygroscopic porous textile may exist in vapor or liquid form in the pore spaces. Phase changes associated with water include liquid evaporation/condensation in the pore spaces and sorption/desorption from polymer fibers. Additional factors such as swelling of solid polymer due to water and heat of sorption was incorporated into the appropriate conservation and transport equations. Nordon and David [9] have attempted to solve the non-linear differential equations which describe coupled diffusion of heat and mass (moisture) in hygroscopic textile materials. In addition to the diffusion equations, a rate equation was introduced describing the rate of exchange of moisture between the solid (textile fibers) and the gas phase. The predictions compared favorably with experimental observations on wool bales and wool fabrics [10]. Farnworth [11] has developed a simple (algebraic) model of combined heat and water vapor transport in clothing. Transport by forced convection was not included in this model. The calculations were performed in a time dependent mode and compared to experiments performed on a sweating hot plate in a nonsteady state mode. Much of this work [8]-[11] was performed for single layers of cotton or wool fabrics and is not directly relevant to fire fighter protective clothing. Bamford and Boydell [12] have developed a finite difference burn injury evaluation code (ICARUS) for evaluating time to seconddegree burns caused by thermal radiation. ICARUS models the heat transfer between the clothing layers with associated air gaps and a multilayered skin model. The model does not account for moisture transport through the protective clothing. 
Recently, the Building and Fire Research Laboratory (BFRL) at the National Institute of Standards and Technology (NIST) has developed a physics-based computational model for predicting the thermal performance of dry fire fighters protective clothing [13]. This model successfully predicted heat transfer through clothing and provided insight into how the garment would respond to radiative heat flux exposures. This first step in the development of predictive methods for protective clothing helped to advance the science and holds promise, but is only one of the many elements required for accurate prediction of thermal performance across the broad use range of these garment systems. In this work, we extend the model to study the thermal performance of fire fighters' protective clothing under wet conditions. Section 3 gives a detailed discussion of the mathematical formulation for transient heat and moisture transfer through porous clothing. This model accounts for variation in thermodynamic and transport properties due to the presence of moisture on the fabric. A simplified form of the equations are derived by making assumptions appropriate for fire fighter protective clothing (Section 4). The thermal radiation model is briefly discussed in Section 5, followed by the the numerical implementation of the model. A brief section has been included to discuss the initial, boundary and interface conditions. Numerical simulation results are reported for a wet thermal liner in Section 7. These results are compared with experimental data. The results have been analyzed to obtain a detailed understanding of the various physical processes. Finally, we discuss the conclusions (Section 8) that we have derived from this project and our detailed recommendations for future research activities (Section 9).

\section{Detailed Model of Transient Heat and Moisture Transfer Through Cloth}

Before proceeding to formulate the present problem of heat and moisture transfer, when the outer layer of cloth is irradiated by high intensity thermal radiation, several important assumptions can be made to simplify the formulation

- Mass transfer of moisture is caused by molecular diffusion and bulk flow of vapor through the gas volume surrounding the cloth fibers (inter-fiber void space), but not by diffusion on the surface or through pores in the cloth fibers (capillary action).

- The local moisture content (moisture regain) of the cloth is at all times in equilibrium with the partial pressure of water vapor at the temperature of the local gas in the voids. It is assumed that the rate of equilibration is much faster than the vapor diffusion process.

- Volume changes of the fibers due to the changing moisture content are neglected.

- Heat and mass transport are one-dimensional, and is along the thickness of the fabric layers. There is no lateral transport.

Consider a small volume element of a slab of homogeneously packed textile material of unit area and of thickness $\Delta X$. The equation for conservation of mass and energy will be expressed in terms of unit volume of the textile-air mixture. Water vapor is free to convect and diffuse through the interfiber void space and to be sorbed or desorbed by the fibers. Water vapor can accumulate in the unit element both in the void space and in the fibers. The total mass balance continuity equation for water vapor species (gas and liquid phase) can be written as

$$
\frac{\partial}{\partial t}\left[\frac{\rho_{o}(1-f) M}{W_{1}}\right]+\frac{\partial}{\partial t}\left[f C X_{1}\right]=-f \frac{\partial}{\partial x}\left(C v^{*} X_{1}\right)-f \frac{\partial}{\partial x}\left[-\mathcal{D}_{12} C \frac{\partial X_{1}}{\partial x}\right],
$$

where 


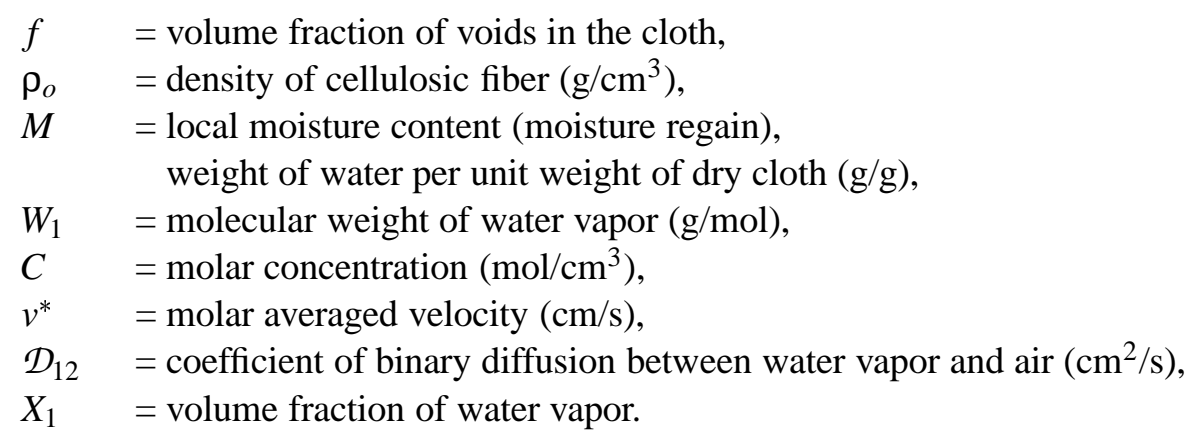

The first term on the left hand side represents the accumulation of water in the fibers. Note that $\rho_{o}(1-f)$ is the dry density of the cloth and is different from $\rho_{o}$, the density of the cellulosic fiber. The second term on the left hand side represents the accumulation of water vapor in the inter-fiber void space. The accumulation of water inside the control volume can change due to bulk flow of vapor (first term on the right hand side) and molecular diffusion (second term on the right hand side) through the gas volume surrounding the cloth fibers.

Similarly the continuity equation for air can be written as

$$
\frac{\partial}{\partial t}\left[f C X_{2}\right]=-f \frac{\partial}{\partial x}\left(C v^{*} X_{2}\right)-f \frac{\partial}{\partial x}\left[-\mathcal{D}_{21} C \frac{\partial X_{2}}{\partial x}\right]
$$

where, $X_{2}$ is the volume fraction of air. Note, that subscript 1 refers to water vapor and subscript 2 refers to air. Equations 1 and 2 have to be solved subject to the constraint equation, that the sum of the volume fraction is equal to one $\left(X_{1}+X_{2}=1\right)$.

Conservation of energy for the control volume can be stated as

$$
\begin{aligned}
\frac{\partial}{\partial t}\left[\frac{\rho_{o}(1-f) M H_{1}}{W_{1}}\right] & +\frac{\partial}{\partial t}\left[\rho_{o}(1-f) H_{c}\right]+\frac{\partial}{\partial t}\left[f C\left(X_{1} h_{1}+X_{2} h_{2}\right]=\right. \\
& -\frac{\partial}{\partial x}\left(-\left(k_{c}+\left(k_{w}-k_{c}\right) f^{\prime}\right) \frac{\partial T}{\partial x}\right)-f \frac{\partial}{\partial x}\left(C v^{*}\left[X_{1} h_{1}+X_{2} h_{2}\right]\right) \\
& -f \frac{\partial}{\partial x}\left[-\mathcal{D}_{12} C \frac{\partial X_{1}}{\partial x} h_{1}-\mathcal{D}_{21} C \frac{\partial X_{2}}{\partial x} h_{2}\right]-\frac{\partial q_{R}}{\partial x},
\end{aligned}
$$

where $^{1}$,

$$
\begin{array}{ll}
H_{1} & =\text { Molal Enthalpy of liquid water }(\mathrm{erg} / \mathrm{mol}), \\
H_{c} & =\text { Enthalpy of fabric }(\mathrm{erg} / \mathrm{g}), \\
h_{1} & =\text { Molal Enthalpy of water vapor }(\mathrm{erg} / \mathrm{mol}), \\
h_{2} & =\text { Molal Enthalpy of air }(\mathrm{ergs} / \mathrm{mol}), \\
k_{c} & =\text { Thermal Conductivity of fabric }(\mathrm{erg} / \mathrm{cm} / \mathrm{s} / \mathrm{K}), \\
k_{w} & =\text { Thermal Conductivity of liquid water }(\mathrm{erg} / \mathrm{cm} / \mathrm{s} / \mathrm{K}), \\
f^{\prime} & =\text { Volume fraction of liquid water, } \\
q_{R} & =\text { Radiative Flux. }
\end{array}
$$

The left hand side of the energy equation is the rate of accumulation of enthalpy in the control volume. The first term on the left hand side is the contribution due to change in enthalpy of liquid water, the second term is

\footnotetext{
${ }^{1}$ The policy of the National Institute of Standards and Technology is to use SI units of measurement in all its publications. In this document, however, the CGS system of units is sometimes used because of the scales and ranges of the quantities and the wide use of such units in the fire modeling field. For clarification, erg $=10^{-7} \mathrm{~J}$, dyne $=10^{-5} \mathrm{~Pa}$.
} 
due to the fabric and the last term is due to water vapor air mixture that is present in the control volume. The first term on the right hand side (Equation 2) shows the net rate of heat addition due to thermal conduction into the control volume. The second term on the right hand side represents the net rate of influx of enthalpy by convection, while the third term shows contribution due to molecular diffusion in the inter-fiber void space. The last term on the right hand side of Equation 2, represents the net rate of heat added due to the incident radiative flux on the control volume. This term will be discussed in more detail in section 5 .

Since $f^{\prime}$, the volume fraction of liquid water in the control volume is equal to $\rho_{o}(1-f) M / \rho_{w}$, the total thermal conductivity $k_{T}$ can be defined as

$$
k_{T}=k_{c}+\left(k_{w}-k_{c}\right)\left[\frac{\rho_{o}(1-f) M}{\rho_{w}}\right],
$$

where, $\rho_{w}$ is the density of the liquid water $\left(\mathrm{g} / \mathrm{cm}^{3}\right)$. The total thermal conductivity is a volume weighted measure of thermal conductivity of cloth and of liquid water. Thermal conductivity of water is 23 times that of air, so the contribution to $k_{T}$ from air and water vapor are assumed to be negligible. Thus, the total thermal conductivity increases linearly with moisture regain $M$, in agreement with experiments by Schneider and Hoschke [14].

To progress any further, we must somehow relate the moisture content $M$ of the fabric to the water vapor volume fraction $X_{1}$ in the gas phase. If the gas flows slowly through the pores, then the cloth and gas phase will stand in approximate local equilibrium at all times. This assumption [15] allows us to neglect the detailed kinetics of adsorption in favor of standard equilibrium thermodynamics, and to assume a definite functional relationship such as

$$
M=\Phi\left(T, X_{1}\right) .
$$

This relationship is known as an adsorption isotherm [16] and it relates the moisture content $M$ to water vapor volume fraction $X_{1}$, and is a function of temperature [17], [18]. The adsorption isotherm was measured experimentally for a few fire fighter protective clothing fabrics using the HYDEN Adsorption Analyzer ${ }^{2}$.

Finally, we close the system of equations using the equation of state

$$
P=C R_{o} T \text {, }
$$

where, $R_{o}$ is the Universal gas constant $\left(8.314 \times 10^{7} \mathrm{erg} / \mathrm{mol} / \mathrm{K}\right)$.

Using equations 1, 2, 3, 5 and 6 along with constraint equations and appropriate initial and boundary conditions, one can obtain a solution of temperature $T$, moisture regain $M$, the volume fractions $X_{1}$ and $X_{2}$ and the molar density $C$ as a function of distance $x$ and time $t$. The mathematical solution of the set of equations based on a realistic model of movement of water vapor, by molecular diffusion and bulk flow, through cloth, when subjected to an external radiation source, is challenging. The complexity of the problem, arises from the fact that there is no simple way to evaluate the bulk flow velocity of water vapor through the inter-fiber void spaces, when the cloth is heated to over $100{ }^{0} \mathrm{C}$ in a very short time (fraction of a second), at very high radiation intensity. Instead of solving the Navier-Stokes equations in the void spaces, the bulk flow velocity can be calculated simply from the local gradient in total pressure of the system and can be expressed using Darcy's momentum equation

$$
v^{*}=\frac{K}{\mu}\left(\frac{\partial P}{\partial x}\right)
$$

where,

\footnotetext{
${ }^{2}$ Certain commercial equipment, instruments, or materials are identified in this paper in order to adequately specify the materials used and the experimental procedure. Such identification does not imply recommendation or endorsement by the National Institute of Standards and Technology, nor does it imply that the materials or equipment identified are necessarily the best available for the purpose.
} 


$$
\begin{array}{ll}
P & =\text { Total Pressure }\left(\mathrm{dyn} / \mathrm{cm}^{2}\right), \\
\mu & =\text { Fluid viscosity } \\
K & =\text { Fabric permeability }\left(\mathrm{cm}^{2}\right) .
\end{array}
$$

However, at very high radiation intensities, the flow of vapor due to the total pressure gradient will exceed that transferred by diffusion and a theory based solely on diffusion will break down under those conditions. Note, that Darcy's momentum equation provides a linear relationship that is characteristic of Stokes flow (viscous flow domination over inertial force) and is not valid at high velocities. More importantly, the external flow field generated by the fire which surrounds the fire fighter must be known a priori, and imposed as a boundary condition on the present analysis (which is limited to the fabric and air gaps). Experiments have also shown that at moderately high radiation intensities, fabrics in contact with skin cannot withstand more than a few second exposure without being scorched. Any theory that is expected to describe such a situation adequately must also include the effect of cloth decomposition or chemical reaction, which is still beyond our present ability. Besides, most fire fighter injuries (second degree burns) take place at low to medium incident intensities when there is little or no thermal degradation of their protective gear. We next develop an approximate model of heat and mass transfer through porous cloth valid for low to medium intensities, assuming that the gas flows slowly through the cloth and that adsorption isotherms relating moisture content to vapor pressure are valid.

\section{Approximate Model of Transient Heat and Moisture Transfer Through Cloth}

Multiplying equations 1 and 2 by $h_{1}$ and $h_{2}$ respectively, and then adding them together we obtain,

$$
\begin{aligned}
h_{1} \frac{\partial}{\partial t}\left[\frac{\rho_{c} M}{f W_{1}}+C X_{1}\right] & +h_{2} \frac{\partial}{\partial t}\left[C X_{2}\right]=-h_{1} \frac{\partial}{\partial x}\left(C v^{*} X_{1}\right)-h_{2} \frac{\partial}{\partial x}\left(C v^{*} X_{2}\right) \\
& -h_{1} \frac{\partial}{\partial x}\left[-\mathcal{D}_{12} C \frac{\partial X_{1}}{\partial x}\right]-h_{2} \frac{\partial}{\partial x}\left[-\mathcal{D}_{21} C \frac{\partial X_{2}}{\partial x}\right]
\end{aligned}
$$

where, $\rho_{c}=\rho_{o}(1-f)$ is the dry density of the fabric. Subtracting equation 8 from equation 3 , we obtain

$$
\begin{aligned}
\left(H_{1}-h_{1}\right) \frac{\partial}{\partial t}\left[\frac{\rho_{c} M}{W_{1}}\right] & +\left[\frac{\rho_{c} M}{W_{1}}\right] \frac{\partial H_{1}}{\partial t}+\frac{\partial}{\partial t}\left[\rho_{c} H_{c}\right]+f C\left[X_{1} \frac{\partial h_{1}}{\partial t}+X_{2} \frac{\partial h_{2}}{\partial t}\right]= \\
& -\frac{\partial}{\partial x}\left(-k_{T} \frac{\partial T}{\partial x}\right)-f C v^{*}\left[X_{1} \frac{\partial h_{1}}{\partial x}+X_{2} \frac{\partial h_{2}}{\partial x}\right] \\
& +f \mathcal{D}_{12} C\left[\frac{\partial X_{1}}{\partial x} \frac{\partial h_{1}}{\partial x}+\frac{\partial X_{2}}{\partial x} \frac{\partial h_{2}}{\partial x}\right]-\frac{\partial q_{R}}{\partial x} .
\end{aligned}
$$

The enthalpies can be defined as follows

$$
\begin{array}{rll}
h_{1}=c_{1} T & , \quad h_{2}=c_{2} T, \\
H_{1}=C_{1} T+\Delta H^{*} & , \quad H_{c}=C_{c} T,
\end{array}
$$

where,

$$
\begin{array}{ll}
c_{1} & =\text { molal heat capacity of water vapor }(\mathrm{erg} / \mathrm{mol} / \mathrm{K}), \\
c_{2} & =\text { molal heat capacity of air }(\mathrm{erg} / \mathrm{mol} / \mathrm{K}), \\
C_{1} & =\text { molal heat capacity of liquid water }(\mathrm{erg} / \mathrm{mol} / \mathrm{K}), \\
C_{c} & =\text { mass heat capacity of fabric }(\mathrm{erg} / \mathrm{g} / \mathrm{K}), \\
\Delta H^{*} & =\text { Heat of Adsorption }(\mathrm{erg} / \mathrm{mol}) .
\end{array}
$$


Substituting the above in equation 9, and assuming that the heat capacities are independent of temperature, we obtain

$$
\begin{aligned}
{\left[\left(\Delta H^{*}+\left(C_{1}-c_{1}\right) T\right) \frac{\rho_{c}}{W_{1}}\right] \frac{\partial M}{\partial t} } & +\left[\left(\frac{\rho_{c} M C_{1}}{W_{1}}\right)+\rho_{c} C_{c}+f C\left(X_{1} c_{1}+X_{2} c_{2}\right)\right] \frac{\partial T}{\partial t}= \\
& -\frac{\partial}{\partial x}\left(-k_{T} \frac{\partial T}{\partial x}\right)-f C v^{*}\left[X_{1} c_{1}+X_{2} c_{2}\right] \frac{\partial T}{\partial x} \\
& +f \mathcal{D}_{12} C\left[\frac{\partial X_{1}}{\partial x} c_{1}+\frac{\partial X_{2}}{\partial x} c_{2}\right] \frac{\partial h_{2}}{\partial x}-\frac{\partial q_{R}}{\partial x} .
\end{aligned}
$$

In order to derive the approximate model for transient heat and moisture transfer through cloth, we make an additional assumption that, sensible heat transfer in the vapor phase is negligible compared with heat conduction through the cloth and with the latent heat effects of adsorption and desorption of water on the cloth fibers. With this assumption, the energy conservation equation (Eq. 11) simplifies to

$$
\rho_{c} \Delta H \frac{\partial M}{\partial t}+\left[\left(\frac{\rho_{c} M C_{1}}{W_{1}}\right)+\rho_{c} C_{c}\right] \frac{\partial T}{\partial t}=-\frac{\partial}{\partial x}\left(-k_{T} \frac{\partial T}{\partial x}\right)-\frac{\partial q_{R}}{\partial x}
$$

where, $\Delta H=\Delta H^{*} / W_{1}$ is the heat of adsorption per unit mass (erg/g). Furthermore, if we define the total density of the control volume $\rho=\rho_{c}(1+M)$, and the total heat capacity of the control volume $C_{T}=\frac{C_{c}+M C_{1} / W_{1}}{1+M}$, then the energy equation (equation 12) can be expressed simply as

$$
\rho C_{T} \frac{\partial T}{\partial t}=-\frac{\partial}{\partial x}\left(-k_{T} \frac{\partial T}{\partial x}\right)-\frac{\partial q_{R}}{\partial x}-\rho_{c} \Delta H \frac{\partial M}{\partial t} .
$$

The final form of the energy equation (Eq. 13) simply states that the increase in heat content of the material inside the control volume (textile fabric, air, water and water vapor) is equal to the net amount of heat entering by conduction plus the contribution due to radiative heating of the volume plus the heat evolved as water vapor is adsorbed inside the control volume. Note, that the total heat capacity $C_{T}$ is a non-linear function of the moisture content $M$, and has contributions from the heat capacity of dry fabric and that of liquid water. Both thermal conductivity $k_{T}$ and heat capacity increase substantially with moisture regain, however, thermal diffusivity $k_{T} / \rho C_{T}$ shows a minor variation with moisture regain.

Simplification of the mass conservation equation requires an estimation of the bulk flow velocity term. As mentioned earlier, we have restricted our attention to the problem of transient heat and moisture transfer through fabrics subjected to low or medium radiation intensities. We now assume that the volume of air flowing through the fabric is negligible compared with that of the water vapor due to desorption and adsorption of water to and from the cloth fibers. Air is assumed to be a stagnant layer through which water vapor flows. Thus if the rate of transfer for the air species is set to zero, we obtain

$$
C v^{*} X_{2}-C \mathcal{D}_{12} \frac{\partial X_{2}}{\partial x}=0
$$

Since $X_{1}+X_{2}=1$, we obtain

$$
C v^{*}=-\left[\frac{C \mathcal{D}_{12}}{1-X_{1}}\right] \frac{\partial X_{1}}{\partial x}
$$

Substituting 15 in equation 1, the continuity equation for water can be re-written as

$$
\frac{\rho_{c}}{W_{1}} \frac{\partial M}{\partial t}+f C \frac{\partial X_{1}}{\partial t}=-f \frac{\partial}{\partial x}\left[-\frac{\mathcal{D}_{12} C}{\left(1-X_{1}\right)} \frac{\partial X_{1}}{\partial x}\right]
$$


Replacing volume fraction $X_{1}$ by fractional partial pressure $p / P$ we obtain

$$
\frac{\rho_{c}}{f} \frac{\partial M}{\partial t}=-\frac{\rho_{g} W_{1}}{P \bar{W}} \frac{\partial p}{\partial t}-\frac{\partial}{\partial x}\left[\left(-\frac{W_{1} \mathcal{D}_{12}}{R_{o} T(1-p / P)}\right) \frac{\partial p}{\partial x}\right],
$$

where, $p$ is the vapor pressure and $\rho_{g}$ is the gas density $\left(\mathrm{g} / \mathrm{cm}^{3}\right)$ defined as

$$
\rho_{g}=C \bar{W}=P \bar{W} /\left(R_{o} T\right)
$$

$\bar{W}$ is the mean molecular weight of the system. If we further define the mass diffusion coefficient, $D$ as

$$
D=\frac{\mathcal{D}_{12} W_{1}}{R_{o} T(1-p / P)}
$$

then the final form of the mass continuity equation can be re-written as

$$
\frac{\rho_{c}}{f} \frac{\partial M}{\partial t}=-\frac{\rho_{g} W_{1}}{P \bar{W}} \frac{\partial p}{\partial t}-\frac{\partial}{\partial x}\left[-D \frac{\partial p}{\partial x}\right]
$$

The mass continuity equation (Eq. 20) states that the net amount of vapor entering the control volume by diffusion is equal to the increase in moisture in air plus the increase in the moisture content of the fibers. The mass continuity equation (Eq. 20), the energy conservation equation (Eq. 13) along with the equilibrium relationship (Eq. 5) are numerically solved in each fabric layer and air gap to obtain the three unknown variables (moisture content $M$, temperature $T$ and vapor pressure $p$ ). Note, that the equilibrium relationship can be easily expressed in terms of vapor pressure $p$, instead of the the volume fraction $X_{1}$. The gas density $\rho_{g}$ is obtained from the equation of state 18. The numerical techniques used to solve the equations are discussed in Section 6.

\section{Thermal Radiation Model}

To obtain the temperature distribution throughout the protective clothing ensemble both the energy equation (Eq. 13) and the radiative heat transfer equation (in some approximation) must be solved. The thermal radiation model described here, is an extension of the model developed by Mell and Lawson [13] for fire fighter protective clothing. We assume, that within a material layer, the contribution to radiative flux from self emission is much smaller than that due to the absorption of the externally incident flux. The major radiative flux incident on the boundaries of a material layer are assumed to be due to :

1. the external incident heat flux on the outer garment layer. This contributes to the forward incident fluxes and the backward incident fluxes on the inner gas/solid boundaries, if reflection occurs.

2. interlayer (across air layers) radiative flux and its reflection. This occurs when the material surfaces bounding the air layers have different temperatures and contributes to both the forward and backward incident flux.

With regards to 1 , only the backward reflection of radiation due to the external flux is calculated. Also in the present model, the net flux, which is found by integrating over all the wavelengths is considered [19]. Under these assumptions, the net radiative flux $q_{x, l}$ at a distance $x$ measured from the left boundary, within material layer $l$ can be obtained from the Beer-Lambert law, and can be expressed as

$$
q_{x, l}=q_{l}^{i+} e^{-\kappa_{l} x}+q_{l}^{i-} e^{-\kappa_{l}\left(d_{l}-x\right)},
$$




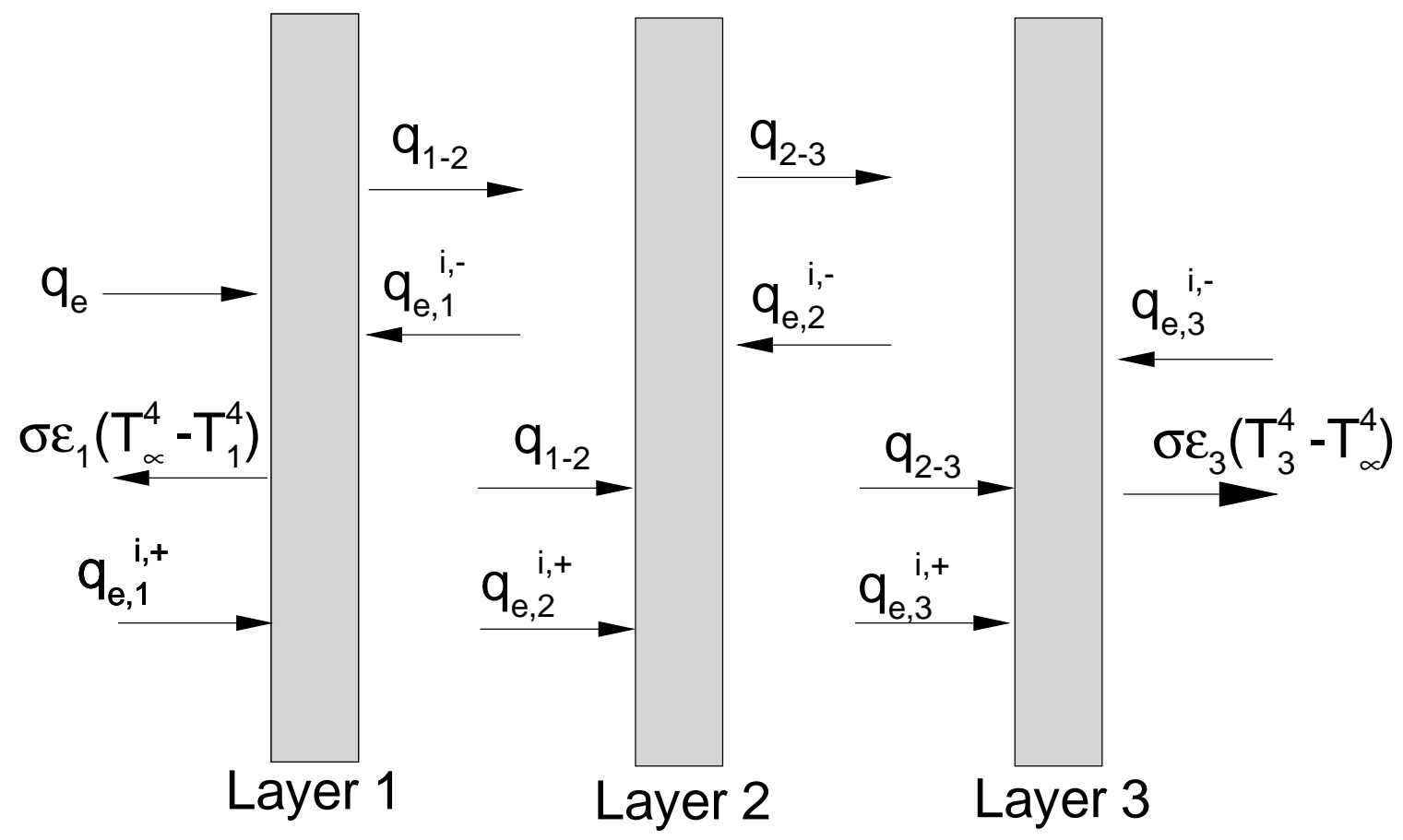

Figure 1: Schematic cross-section of a three layered firefighter protective clothing ensemble surrounded by ambient air. Radiative fluxes incident on material boundaries have been indicated.

where, $q_{l}^{i+}$ and $q_{l}^{i-}$ are the fluxes incident on the left and right side, respectively, of material layer $1, \kappa_{l}$ is the absorption coefficient of layer $l$ and $d_{l}$ is the thickness of material layer $l$.

The absorptivity of a fabric layer, $\alpha$, is related to it transmissivity, $\tau$, and reflectivity $r$, through

$$
\alpha+r+\tau=1 .
$$

The absorption coefficient $\kappa_{l}$ is assumed constant within the material layer $l$, and can be determined from the transmissivity and reflectivity of the fabric layer as

$$
\kappa_{l}=\frac{1}{d_{l}} \ln \left(\frac{1-r_{l}}{\tau_{l}}\right)
$$

where, $d_{l}$ is the thickness of the fabric layer $l$.

The fluxes incident on the left and right hand side of the material boundary are from two sources: the external radiation source $\left(q_{e}\right)$ and the interlayer flux denoted as $q_{1-2}$ for fabric layers 1 and 2 . These fluxes have been depicted in Figure 1 for a three layered clothing assembly surrounded by ambient air of temperature $T_{\infty}$. 
The contribution to the incident boundary flux due to the external flux are as follows:

$$
\begin{aligned}
& \text { Fabric Layer } 1 \\
& q_{e, 1}^{i+}=q_{e}\left(1-r_{1}\right) \\
& q_{e, 1}^{i-}=-q_{e} r_{2}\left(1-r_{1}\right) e^{\left(-\kappa_{1} d_{1}\right)}
\end{aligned}
$$

Fabric Layer 2

$q_{e, 2}^{i+}=q_{e}\left(1-r_{2}\right)\left(1-r_{1}\right) e^{\left(-\kappa_{1} d_{1}\right)}$

$q_{e, 2}^{i-}=-q_{e} r_{3}\left(1-r_{2}\right)\left(1-r_{1}\right) e^{\left(-\kappa_{1} d_{1}-\kappa_{2} d_{2}\right)}$

Fabric Layer 3

$q_{e, 3}^{i+}=q_{e}\left(1-r_{3}\right)\left(1-r_{2}\right)\left(1-r_{1}\right) e^{\left(-\kappa_{1} d_{1}-\kappa_{2} d_{2}\right)}$

$q_{e, 3}^{i-}=0$

The net interlayer radiative flux is computed using the result for plane parallel plates with isotropic scattering, diffusively reflecting boundaries separated by nonparticipating air [13]. With this assumption $q_{1-2}$ integrated over all wavelengths is

$$
q_{1-2}=\frac{\sigma\left(\varepsilon_{1} T_{1}^{4}+r_{1} \varepsilon_{2} T_{2}^{4}\right)}{1-r_{1} r_{2}}-\frac{\sigma\left(\varepsilon_{2} T_{2}^{4}+r_{2} \varepsilon_{1} T_{1}^{4}\right)}{1-r_{1} r_{2}}
$$

Thus, the net incident flux on the the left side of a material layer $l=2$ (for example) is the sum of the two contributions

$$
q_{2}^{i,+}=q_{e, 2}^{i,+}+q_{1-2} .
$$

This incident flux is used in equation 21 for computing the net radiative flux and subsequently in the energy equation 13 .

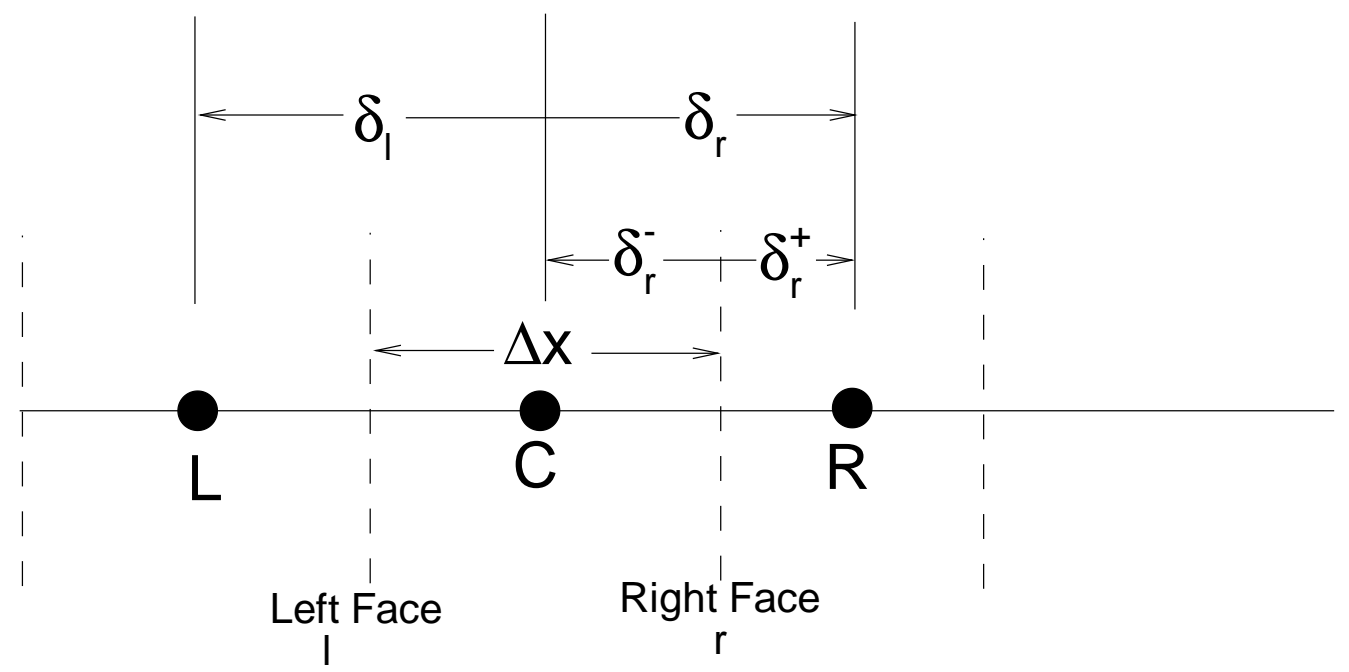

Figure 2: Grid spacing and dimensions for a one-dimensional control volume used in the model for fire fighter protective clothing. 


\section{Numerical Model}

The partial differential equations expressing conservation of energy (Eq. 13) and the mass continuity equation (Eq. 20) derived in the previous section were discretized using a control volume approach to derive the finite difference form of the equations. The method of discretization ensures local energy and mass conservation and requires approximation of only first order derivatives (rather than higher order derivatives).

An arbitrary control volume surrounding grid point $\mathrm{C}$ (at the center of the control volume is shown in Figure 2. Control volume faces are marked by dashed lines, the left face is denoted by 1 and the right face by $\mathrm{r}$. Note that the material interfaces are at the control volume interfaces. Grid points to the left and right of $\mathrm{C}$ are denoted by $\mathrm{L}$ and $\mathrm{R}$, respectively. The control volumes centered around $\mathrm{C}, \mathrm{L}$ and $\mathrm{R}$ may not be of the same size, i.e.

$$
\delta_{l} \neq \delta_{r} \neq \delta x
$$

The goal of the numerical procedure is to predict the temperature $\mathrm{T}$, vapor presure $\mathrm{P}$ and moisture content $\mathrm{M}$ at the cell centers. It is assumed that these quantities vary linearly within the control volume. The coefficient of the various terms are computed at the cell center and are assumed to be constant within each cell.

Integrating equation 13 over the control volume centered around $\mathrm{C}$ gives

$$
\int_{l}^{r} \rho C_{T} \frac{\partial T}{\partial t} d x d A=\left.k_{T} \frac{\partial T}{\partial x}\right|_{l} ^{r} d A-\left.q_{R}\right|_{l} ^{r} d A-\int_{l}^{r} \rho_{c} \Delta H \frac{\partial M}{\partial t} d x d A
$$

The first order derivates in the conduction flux term are obtained by assuming that $\mathrm{T}$ varies linearly with the control volume. Care is taken to ensure that the flux is continuous at the cell interfaces. Similarly, integrating equation 20 results in

$$
\int_{l}^{r} \frac{\rho_{c}}{f} \frac{\partial M}{\partial t} d x d A=-\int_{l}^{r} \frac{\rho_{g} W_{1}}{P \bar{W}} \frac{\partial p}{\partial t} d x d A+\left.D \frac{\partial p}{\partial x}\right|_{l} ^{r} d x d A
$$

The discretized form of the above equations are solved for each material layer and air gap. A second order explicit Runge-Kutta scheme was used for time stepping [13]. Although the stability characteristics of these methods are more restrictive than implicit methods, explicit Runge-Kutta techniques result in more accurate solutions than implicit schemes of comparable order.

\subsection{Initial Conditions, Boundary Condition and Interface Conditions}

A unique solution to the governing equations requires the specification of initial and boundary conditions. The initial temperature distribution in the fabric layers and the air gap has to be specified. Normally, room temperature values are specified as measured in the laboratory. The initial moisture distribution inside the fabric layers is also specified as an initial condition. The vapor pressure within the fabric layer is then computed from the equilibrium relationship. Temperature and vapor pressure in the air gap are normally specified at room temperature and relative humidity. The incident radiative flux as a function of time must also be specified to start the calculations.

Boundary conditions must be specified at the edges of the computational domain. This includes specification of temperature and vapor pressure or relative humidity at the right and left edges of the computational domain. Besides specification of initial and boundary conditions, specialized interface conditions have to be formulated at the gas / solid boundaries. There are two types of gas / solid boundaries, according to whether or not convective heat and mass transfer is present. It is assumed that the air gaps between the fabric layers are small enough and the velocity boundary layer does not develop fully within the air gaps. The heat transfer in these air gaps and the adjoining material layers is by conduction only while mass transfer is by 
diffusion only. Convective heat and mass transfer are very important at the outer surface of the turnout coat exposed to the ambient atmosphere.

The surface heat transfer coefficient $h_{c}$, was obtained from the Nusselt number through empirical correlation of free convection on a vertical plate [20], [21]

$$
h_{c}=N u \frac{k_{G}}{L},
$$

where, $N u$ is the Nusselt number, $k_{G}$ is the thermal conductivity and $L$ is the characteristic length. Correlations appropriate for both laminar and turbulent flow were used depending on the magnitude of the Rayleigh number. The surface mass transfer coefficient $m_{c}$ was obtained from the heat transfer coefficient $h_{c}$ by assuming that the ratio of Schmidt to Prandtl numbers is unity. Thus,

$$
\frac{h_{c}}{m_{c}}=P C_{\text {vapor }} \frac{M}{M_{w}}
$$

where, $P$ is the total pressure, $C_{\text {vapor }}$ is the specific heat of humid air and $M / M_{w}$ is the ratio of mean molecular weight of gas to that of water. These correlations are obtained from work done by Carslaw and Jaegar [22]. The surface heat and mass transfer coefficients are used in estimating the heat and mass fluxes at the boundaries of the garment as discussed earlier.

\section{Results and Discussion}

In order to demonstrate the effect of moisture on wet fire fighter protective clothing, a series of simulations and experiments were performed. We next describe these simulations and compare them with available experimental data.

The presence of moisture in the fabric and the amount of moisture affects the local density, and thermodynamic properties (heat capacity) as well as transport properties (thermal conductivity and diffusivity). Moisture changes the propagation of the thermal wave into the fabric layer, since some energy is used in heating up the moisture and in the evaporation process. Water vapor can also condense on the fabric and this further affects the temperature distribution. As a result, the moisture distribution inside the fabric layer is an important input quantity for the numerical simulation. Note that we are assuming moisture distribution varies only along the thickness of the fabric layers (one-dimensional problem).

The numerical model is relatively flexible, in that any initial moisture distribution can be specified for each fabric layer as an input from the experimental configuration. Experimentally, determining the moisture distribution within the fabric layers is a challenging task. In the experiments, a known quantity of water was sprayed on the front and back sides of the fabric layers with the hope that this would produce a uniform distribution of moisture in all of the fabric layers. The use of a single fabric layer instead of multiple fabric layer garments, allows us to control and prescribe the moisture content and moisture distribution in a more precise manner. Since detailed comparison of numerical and experimental results require a better understanding of moisture distribution inside the various fabric layers, it was decided to initially restrict this study to single fabric layers. The thermal liner was chosen for this single fabric layer study becuase it absorbs water more readily as compared to the moisture barrier. Also, since the thermal liner is slightly thicker, the thermocouples could be attached more precisely on the front and back faces.

\subsection{Heat and Mass Transfer through a Wet Thermal Liner}

The thermal liner that was used in this analysis is commonly known as ARALITE, manufactured by Southern Mills. The Aralite thermal liner is a needle-bonded Kevlar batting quilted to a dyed aramid face cloth. 


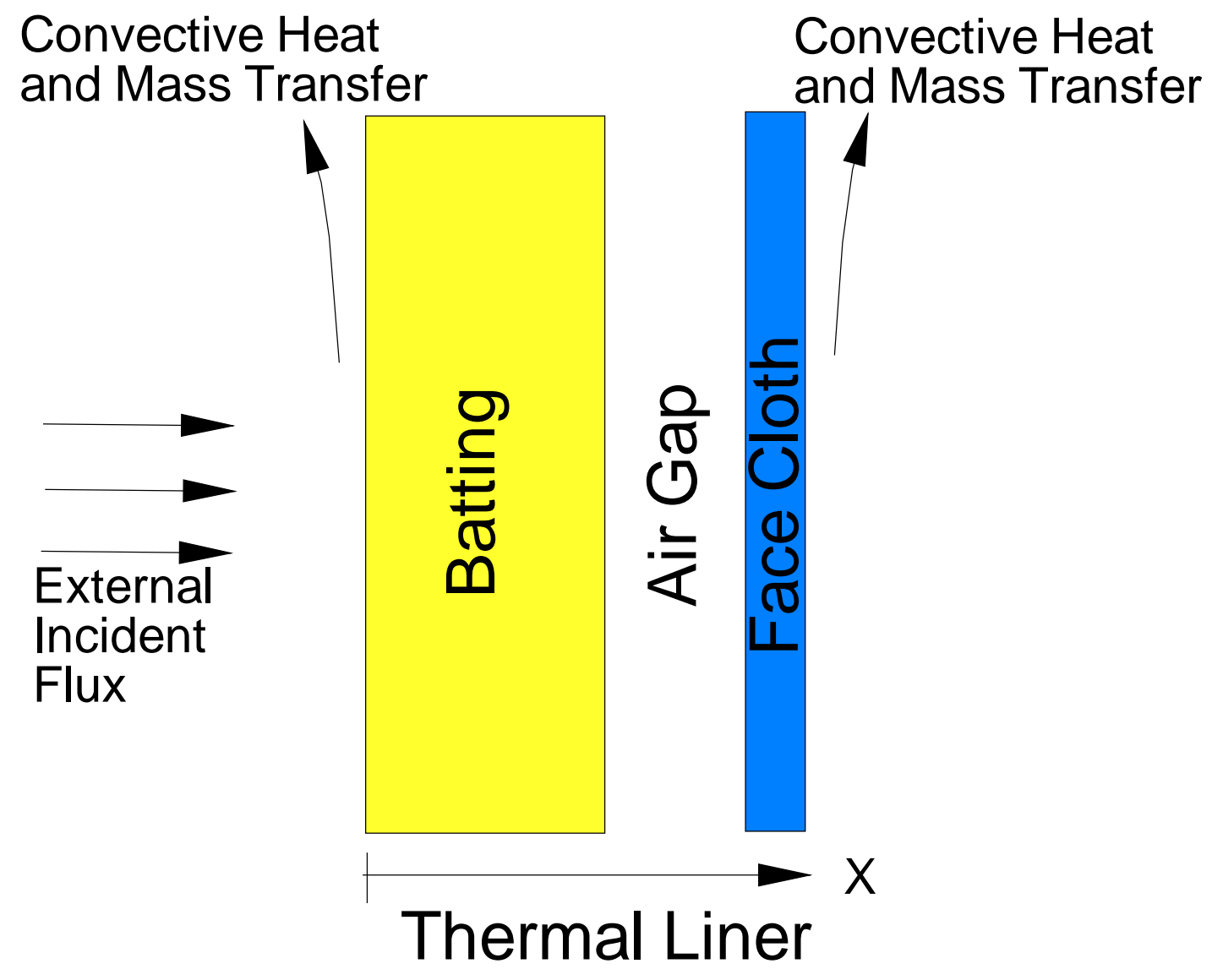

Figure 3: Schematic of an Aralite thermal liner consisting of Kevlar batting quilted to an Aramid face cloth used for studying heat and moisture transfer. The batting and face cloth are $0.265 \mathrm{~cm}$ and $0.025 \mathrm{~cm}$ thick, respectively and are separted by a $0.075 \mathrm{~cm}$ thick air gap.

The open structure of this material provides a high insulation value and is a commonly used fire fighter turnout suit material. To perform a simulation, the thickness, density, conductivity, specific heat and the optical properties of transmissivity and reflectivity were needed. These properties measured and tabulated by Lawson et al. [23] and were used in our calculation.

Figure 3 shows a schematic of the thermal liner consisting of a batting and face cloth. An air gap (1 $\mathrm{mm}$ thick) separates the batting from the face cloth. In the experiments, the batting is subject to an external incident flux of $2.5 \mathrm{~kW} / \mathrm{m}^{2}$ starting at $t=0 \mathrm{~s}$. The external flux is applied until $750 \mathrm{~s}$. The simulation is extended to $850 s$ to allow the fabrics to cool down. A uniform void fraction value of 0.7 for the batting and 0.2 for the face cloth was used. The void fraction is obtained by measuring the compressed and uncompressed (normal loft) thickness of the fabric layer. The thermal liner does not have a clear, planar edge and therefore, thickness measurements in the uncompressed state are difficult. In the compressed state, thickness was found to vary with the pressure and it was unclear if the fiber structure was changed during compression.

In the experiments, two thermocouples are located on the thermal liner. One thermocouple is on the thermal batting and faces the radiant panel. This face is also referred to as the front face or the left face. Another thermocouple is placed on the Aramid face cloth facing away from the radiant panel. This face is referred to as the back face or the right face. Convective heat and mass transfer occur at the front and back 


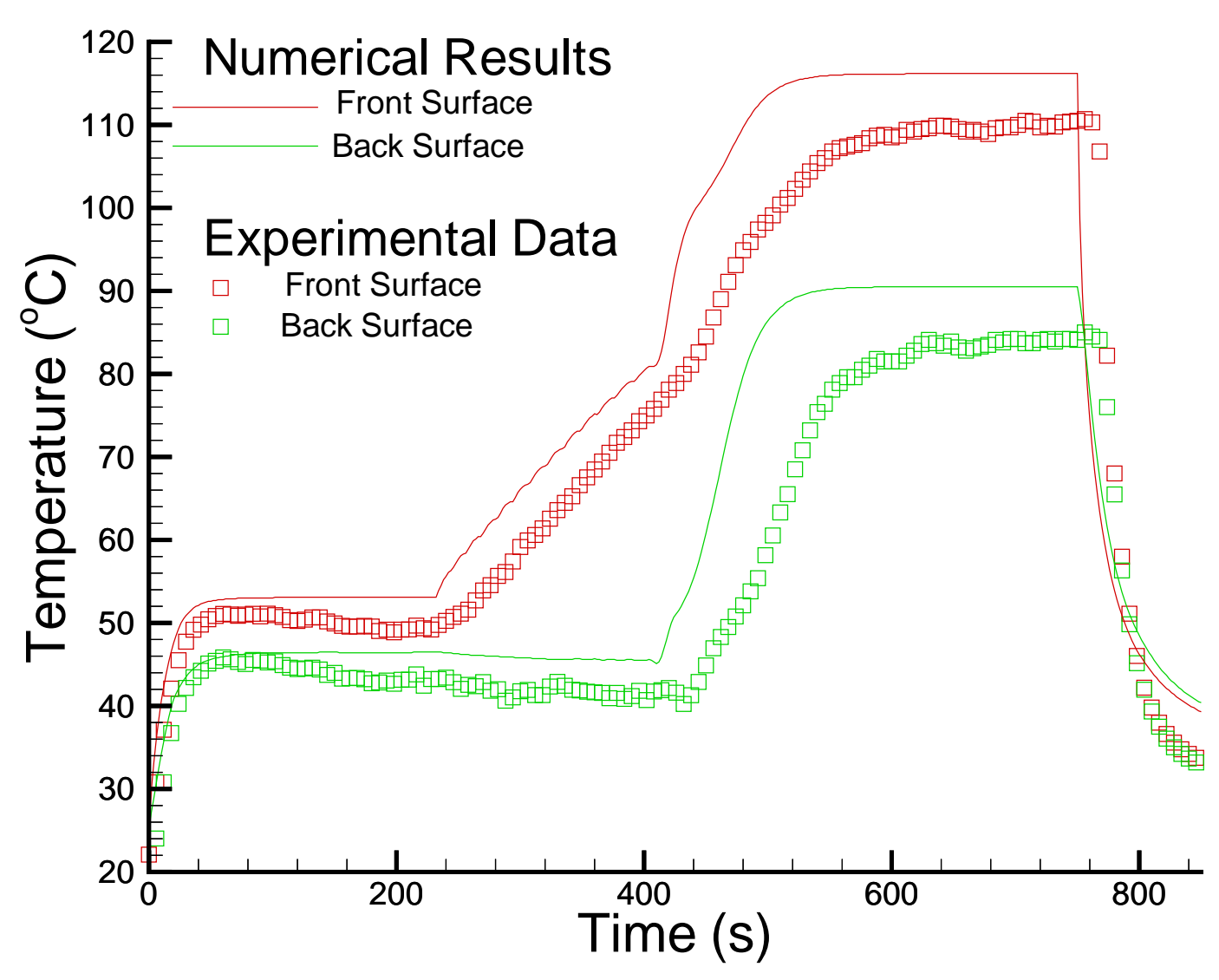

Figure 4: Comparison of numerical and experimental results of temperature history at the front and back faces of the Arlaite thermal liner. The batting is subjected to an incident flux of $2.5 \mathrm{~kW} / \mathrm{m}^{2}$ for $750 \mathrm{~s}$, followed by a cool down period of $100 \mathrm{~s}$.

faces, which are open to the atmosphere. It is assumed that the air gap which is approximately $1 \mathrm{~mm}$ thick does not allow for a fully developed boundary layer. As a result conductive heat transfer is still the more dominant heat transfer mechanism as opposed to convective heat transfer. It is thus assumed in the present analysis that heat and mass transfer between the fabric layer and the air gaps (between the fabric layers) is by diffusive processes alone. Convective heat and mass transfer are active only on the fabric faces that are open to the atmosphere.

Figure 4 shows temperature as a function of time for the front and back faces of the thermal liner when subjected to a radiative heat flux of $2.5 \mathrm{~kW} / \mathrm{m}^{2}$. The numerical results are shown as lines while the experimental data are shown as symbols only. The initial temperature of the fabric is approximately $25^{\circ} \mathrm{C}$. As the thermal liner is exposed to a radiative flux of $2.5 \mathrm{~kW} / \mathrm{m}^{2}$ at $t=0 \mathrm{~s}$, the temperature of the front and back face start rising sharply. The rate of rise for the front face is higher than that for the back face. This is because the front face is exposed directly to the radiant panel. Only a small fraction of this radiant energy is transmitted and heats up the rear face. The numerically predicted profiles follow the experimental data very closely (error is within $5 \%$ ) in this region. Energy is also conducted to the rear face, but this is a relatively slow process. During this initial transient, negligible amount of water has evaporated and the energy is used in heating up the fabric and moisture - air mixture. 
The sharp initial transient is followed by a region where temperature are almost constant. The front face stabilizes at approximately $52{ }^{\circ} \mathrm{C}$ for approximately $200 \mathrm{~s}$, while the back face temperature stabilizes at approximately $45^{\circ} \mathrm{C}$ until $400-420 \mathrm{~s}$. This feature is also observed in the experimental data. In fact, the experimental data show a very slight decrease in temperature. The experimental data shows that the front face temperature reaches approximately $52{ }^{\circ} \mathrm{C}$ and then drops to $50{ }^{\circ} \mathrm{C}$, while the rear face reaches as high as $45^{\circ} \mathrm{C}$ and then cools down to $41-42^{\circ} \mathrm{C}$. In fact the numerical data also indicates a temperature drop of approximately $0.2-0.5^{\circ} \mathrm{C}$, which is difficult to observe on this plot (Figure 4).

The stabilization in temperature indicates that the incident energy is being used to evaporate the moisture. The amount of moisture being evaporated can be computed approximately by dividing the incident energy flux by the heat of evaporation. The length of this plateau region depends on the amount of water in the fabric. If the amount of water on the front face of the fabric is less, the plateau region will be shorter. If there is more water on the front face, the plateau region will be proportionately longer.

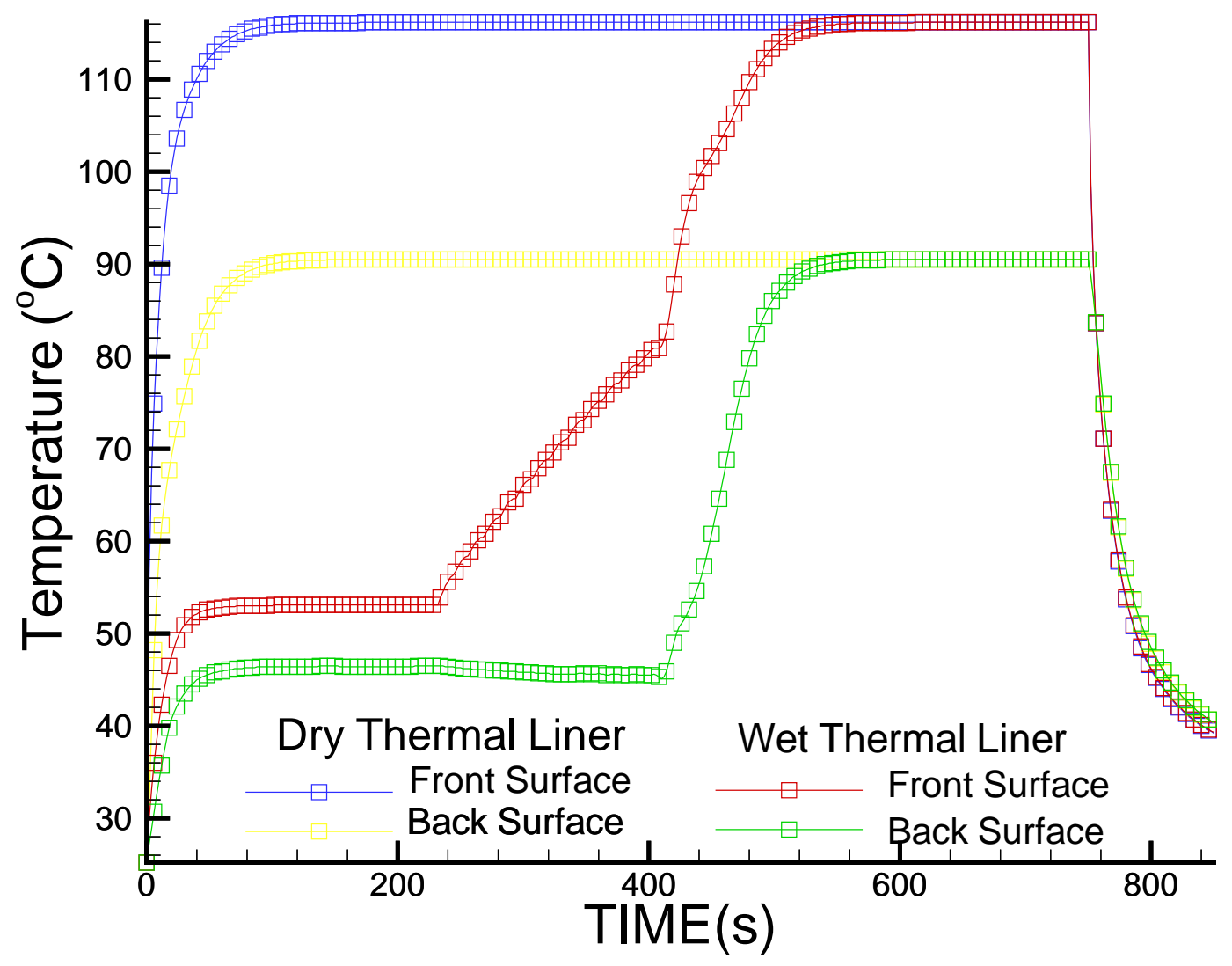

Figure 5: Comparison of numerically predicted temperature histories for the dry and wet thermal liners. For each case, numerical results have been shown for the front face and the back face.

At approximately $200 s$ into the simulation, the temperature of the front face starts rising again, indicating that the front face is completely dry. The back face temperature starts rising at $400-410 s$ indicating that the entire fabric is completely dry. Once the back face is completely dry, the temperature starts rising sharply again until a steady state condition is reached. The experimental results indicate that the temperature does not rise as sharply as the numerical result. The temperature at the back face stabilizes at approximately 


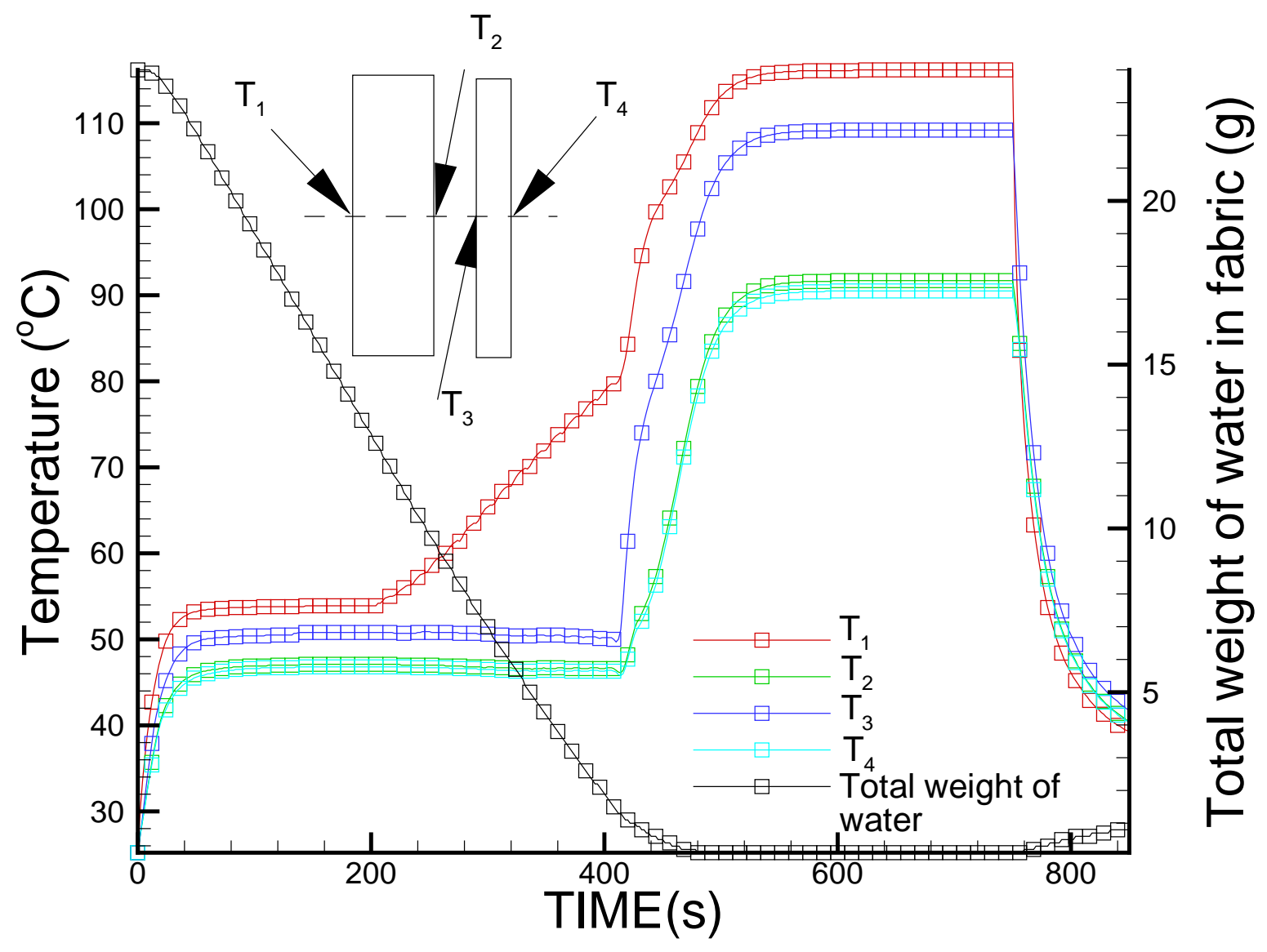

Figure 6: Temperature time histories at the various fabric - air interfaces. The total weight of water (g) in the fabric is plotted as a function of time for the Aralite thermal liner subjected to an incident radiative flux.

$90{ }^{\circ} \mathrm{C}$. The temperature at the front face also starts rising once the front face has completely dried but the rate of rise is relatively small. This rate of temperature increase is smaller than the rate at which the temperature at the back face increases. This rate of temperature increase is also much smaller than the rate of temperature rise from $25{ }^{\circ} \mathrm{C}$ to $50{ }^{\circ} \mathrm{C}$ (see Figure 4) at the front face. At $200 s$ into the simulation, only the front face of the fabric is dry, but there is still water present inside the fabric. The temperature of this portion of the fabric (which is wet) is low which results in conductive heat losses from the front face of the fabric. These conductive losses are higher than what one would expect, if the entire fabric was dry, since the thermal conductively of the wet fabric is higher than that of the dry fabric. Due to these higher conductive heat losses the temperature of the front face rises at a slower rate. In fact the moisture distribution inside the fabric layer can influence the slope of the temperature time curve in this region. Simulations where a different distribution of moisture was assumed resulted in vastly different slopes. The slope of this curve can therefore be used to obtain some information on the moisture distribution inside the fabric. When the entire fabric is completely dry, at $400 s$ into the simulation, the temperature starts rising again at a faster rate. The temperature of the front face stabilizes finally at $115^{\circ} \mathrm{C}$. During the period from $600 s$ to 750 $s$ the temperatures of the front and the back faces remain constant. This indicates that a balance has been reached between the energy flowing into the fabric and convective heat losses at the boundaries. At $t=750$ $s$ into the simulation, the radiative flux from the radiant panel was cut off, which results in a rapid drop in 
temperature of the front and rear faces, consistent with the experimental data.

Our results clearly indicate that there is good qualitative agreement between the numerical predictions and thermocouple measurements.

Figure 5 shows a comparison for a thermal liner that is initially completely dry and a wet thermal liner. For each case, the temperature time curves for the front and back faces of the thermal liner have been shown. The wet thermal liner results are duplicated from Figure 4 for comparison with the dry case. For the dry thermal liner, the temperature at the front face increases sharply from $25{ }^{\circ} \mathrm{C}$ over a period of $30-40 \mathrm{~s}$ and then stabilizes at the steady state value of $115^{\circ} \mathrm{C}$. The temperature of the back face rises less sharply than that of the front face, and this temperature stabilizes at approximately $90{ }^{\circ} \mathrm{C}$. The final temperatures (before the radiative heating is cut-off) are the same for the dry and wet thermal liners. This figure clearly shows the effect of moisture on reducing the temperature at the front and rear faces.

Figure 6 shows the temperatures at all the four interfaces as a function of time. The numerically computed mass loss in grams has also been plotted as a function of time on the $Y_{2}$ axis. The mass loss curve shows the amount of liquid water that is present in the fabric. It is an integrated value over the entire thickness of the fabric. At $t=0 \mathrm{~s}$, the results indicate approximately $24 \mathrm{~g}$ of water. During the first 30 to $40 \mathrm{~s}$ of the simulation, there is very little evaporation of water and the mass loss curve is almost flat. From 40 to $400 \mathrm{~s}$, the total weight of water in the fabric reduces linearly with time. At $t=450 \mathrm{~s}$, all the water has evaporated from the fabric. These observations compared well with the numerically computed temperature histories in the thermal liner. After the radiation has been cut-off, small amounts of water is absorbed back into the fabric, from the surrounding atmosphere. This explains the small increase in mass loss curve after $t=750 \mathrm{~s}$.

\subsection{Time Dependent Simulations}

Figures 7-13 shows time dependent simulation results for a wet thermal liner subjected to an external incident flux. Each figure shows results at a specific time. Each figure has four sub-figures. In the top left sub figure, temperature $\left({ }^{\circ} \mathrm{C}\right)$ as a function of distance has been shown. The distance is measured from the front face of the thermal liner. Dotted lines have been drawn to indicate the location of the interface. The batting material lies between 0.0 and $0.265 \mathrm{~cm}$. The air-gap is between $0.265-0.34 \mathrm{~cm}$ and the face cloth is between $0.34-0.365 \mathrm{~cm}$. The upper right figure shows the vapor pressure $(P)$ in dyn/ $/ \mathrm{cm}^{2}$ as a function of distance. The moisture content $M$ has been shown in the lower left figure while the lower right figure shows the dry fabric density and the wet fabric density as a function of distance.

Figure 7 shows results at time $t=0 \mathrm{~s}$ and thus represent the initial conditions. The temperature is uniform everywhere at $25{ }^{\circ} \mathrm{C}$. The moisture content $M$ shows the assumed moisture distribution in the batting and the face cloth. The resulting density distribution that is initially imposed on the fabric is also shown. The moisture distribution shows a moisture content of 3.0 at the front face. This moisture content drops linearly to a value of 1.5 over the thickness of the batting. The moisture content in the face cloth is set at a constant value of 0.2. In the experiments, water is sprayed on the front and back faces of the fabric. Most of the water sprayed on the front face stays on the front face and we assume a linear decrease in the amount of water as a function of distance. The difference between wet fabric and dry fabric weight distribution clearly illustrates the initial water distribution in the batting. Note that the batting is a relatively thick material with very high void fraction. The water sprayed on the back face is assumed to be uniformly distributed over the face cloth. This distribution of water in the face cloth is reasonable because the face cloth is very thin as compared to the thermal batting.

Figure 8 shows results at $100 s$ into the simulation. The thermal liner is now being radiatively heated

from an external source. The temperature has increased to approximately $50{ }^{\circ} \mathrm{C}$, but is almost uniform over the thickness of the liner. The temperature at the back face is only slightly less than that at the front face. The vapor pressure has increased significantly above its initial value and is close to the saturated 


\section{Time $=0.0$ seconds}
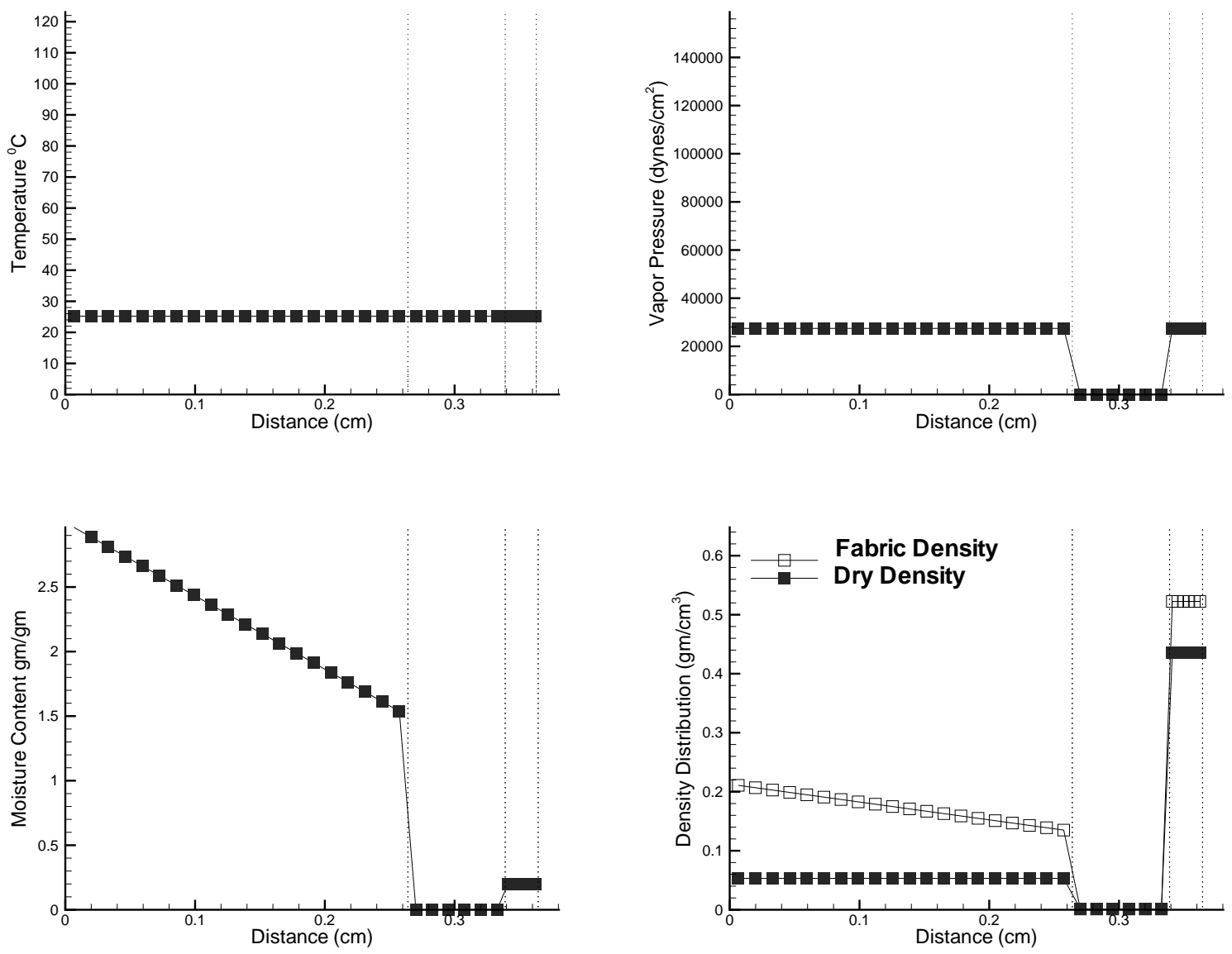

Figure 7: Simulation results at time $t=0 s$ for a wet thermal liner subjected to an external incident flux 2.5 $\mathrm{kW} / \mathrm{m}^{2}$. Temperature (top left sub-figure), vapor pressure (top right sub-figure), moisture content (bottom left sub-figure) and dry / wet density (bottom right sub-figure) has been shown as a function of distance measured from the front face of the thermal liner.

vapor pressure (relative humidity $=100 \%$ ). The moisture content of the fabrics when subjected to a $100 \%$ humidity environment at room temperature is approximately 0.08 . Since the actual moisture content at $t=100 \mathrm{~s}$ is much larger than 0.08 , at each point in the fabric, we expect the vapor pressure to be very close to the saturated vapor pressure. The vapor pressure is higher at the front face and then gradually decreases across the thickness, showing the dependence of vapor pressure on temperature. The moisture content curve indicates that some of the moisture from the front of the fabric has evaporated, which decreases the moisture content. The water vapor that is produced because of the evaporation process can now diffuse towards the front face or towards the back face. The diffusion process depends on the gradient of partial pressure $P$. Also note that convective heat transfer at the front face is continuously removing energy from the fabric. Depending on the vapor pressure of the ambient atmosphere, convective mass transfer can add or remove moisture from the front and rear faces.

Figure 9 shows simulation results at $200 \mathrm{~s}$. The temperature and vapor pressure curves have not changed significantly from their value at $100 s$ (Figure 8). The moisture content curve indicates significant evaporation at the front face. This results in wet fabric density approaching the dry fabric density. We also notice moisture absorbed from the environment at the front face. Higher moisture content levels at the interface 


\section{Time $=100.0$ seconds}
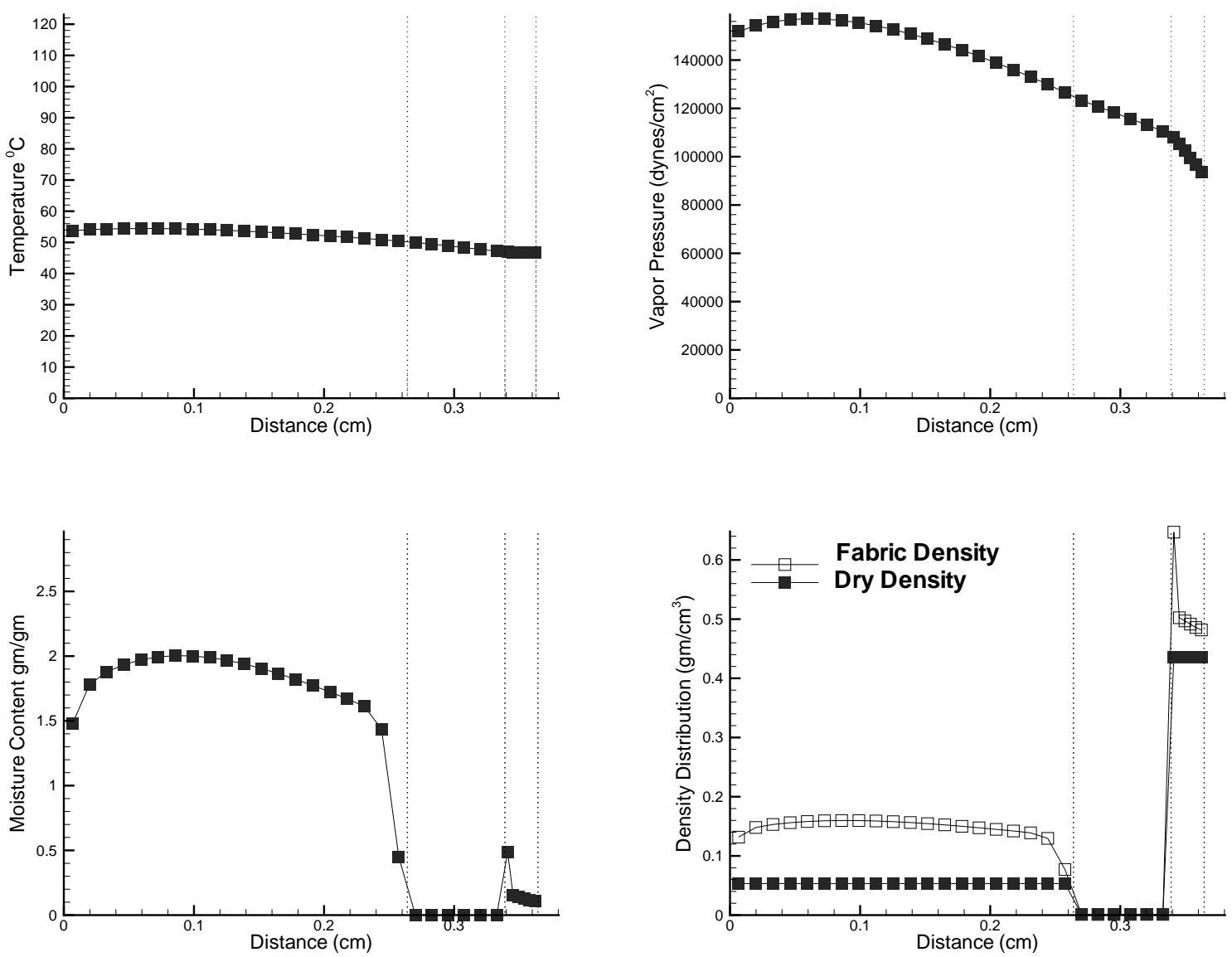

Figure 8: Simulation results at time $t=100 \mathrm{~s}$ for a wet thermal liner subjected to an external incident flux 2.5 $\mathrm{kW} / \mathrm{m}^{2}$. Temperature (top left sub-figure), vapor pressure (top right sub-figure), moisture content (bottom left sub-figure) and dry / wet density (bottom right sub-figure) has been shown as a function of distance measured from the front face of the thermal liner.

between the air gap and the face cloth is indicative of condensation at the face cloth.

At $300 s$ after the onset of external radiation (Figure 10), we start observing an increase in the temperature at the front face to approximately $70{ }^{\circ} \mathrm{C}$. The vapor pressure curve shows a peak at $0.1 \mathrm{~cm}$. The moisture content curve indicates that the front half of the batting material is dry. This is also manifested in the fact that the wet fabric density approaches the dry fabric density. Notice that the peak in the vapor pressure curve coincides with an increase in moisture content. Both these event occur at $0.1 \mathrm{~cm}$. At $400 \mathrm{~s}$ (Figure 11) much of the batting material is essentially dry. The vapor pressure curve shows a peak at 0.22 $\mathrm{cm}$, and the temperature in the batting is beginning to increase at a rapid rate. At $t=500 \mathrm{~s}$ (Figure 12), we observe that the temperature has reached its steady state value everywhere in the thermal liner. The vapor pressure is equal to the ambient pressure and the moisture content is zero at each point in the fabric. The fabric is now completely dry. The density distribution curve indicates that the fabric density is equal to that of the dry fabric.

At $t=750 \mathrm{~s}$, the external radiation source is cut off, and as a result, we observe a rapid drop in temperatures due to convective losses at the boundaries. Because of higher heat capacity of the face cloth (higher density), the temperature of the batting is less than that of the face cloth. We also observe that since the 


\section{Time $=200.0$ seconds}
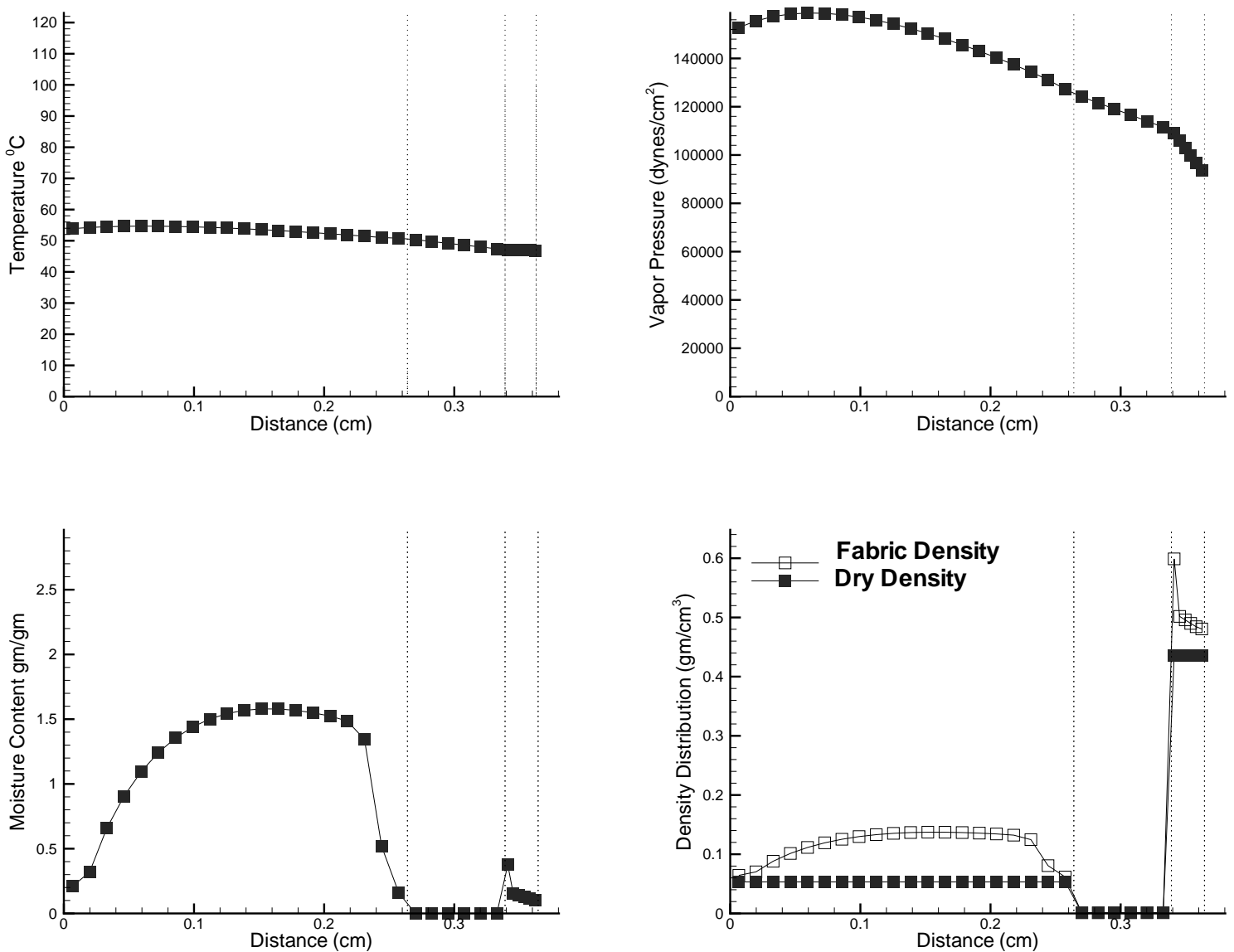

Figure 9: Simulation results at time $t=200 \mathrm{~s}$ for a wet thermal liner subjected to an external incident flux 2.5 $\mathrm{kW} / \mathrm{m}^{2}$. Temperature (top left sub-figure), vapor pressure (top right sub-figure), moisture content (bottom left sub-figure) and dry / wet density (bottom right sub-figure) has been shown as a function of distance measured from the front face of the thermal liner.

vapor pressure is less than the ambient vapor pressure small quantities of water is absorbed at the fabric boundaries due to convective mass transfer.

\subsection{Boundary Fluxes}

Figure 14 shows the convective heat flux at the front surface and the back surface as a function of time. This term is a measure of how rapidly energy is removed from the fabric layers at the outer boundaries. At the front surface, a negative value indicates that the energy flux is in the $-x$ direction implying that energy is being removed from the front face. Similarly at the back face, a positive value indicates that the energy flux is in the $+x$ direction which implies that energy is being removed from the fabric layers. The profile looks very similar to the temperature profiles at the front and back face as shown in Figure 4 . In the case of front surface, the profile is inverted or reflected about the $x$ axis. The convective heat flux increases as the temperature of the fabric increases.

The radiative flux on the front and back surfaces have been shown in figure 15. The radiative flux incident on the front surface is $2.5 \mathrm{~kW} / \mathrm{m}^{2}$. As the front surface becomes heated it also radiates some 


\section{Time $=\mathbf{3 0 0} .0$ seconds}
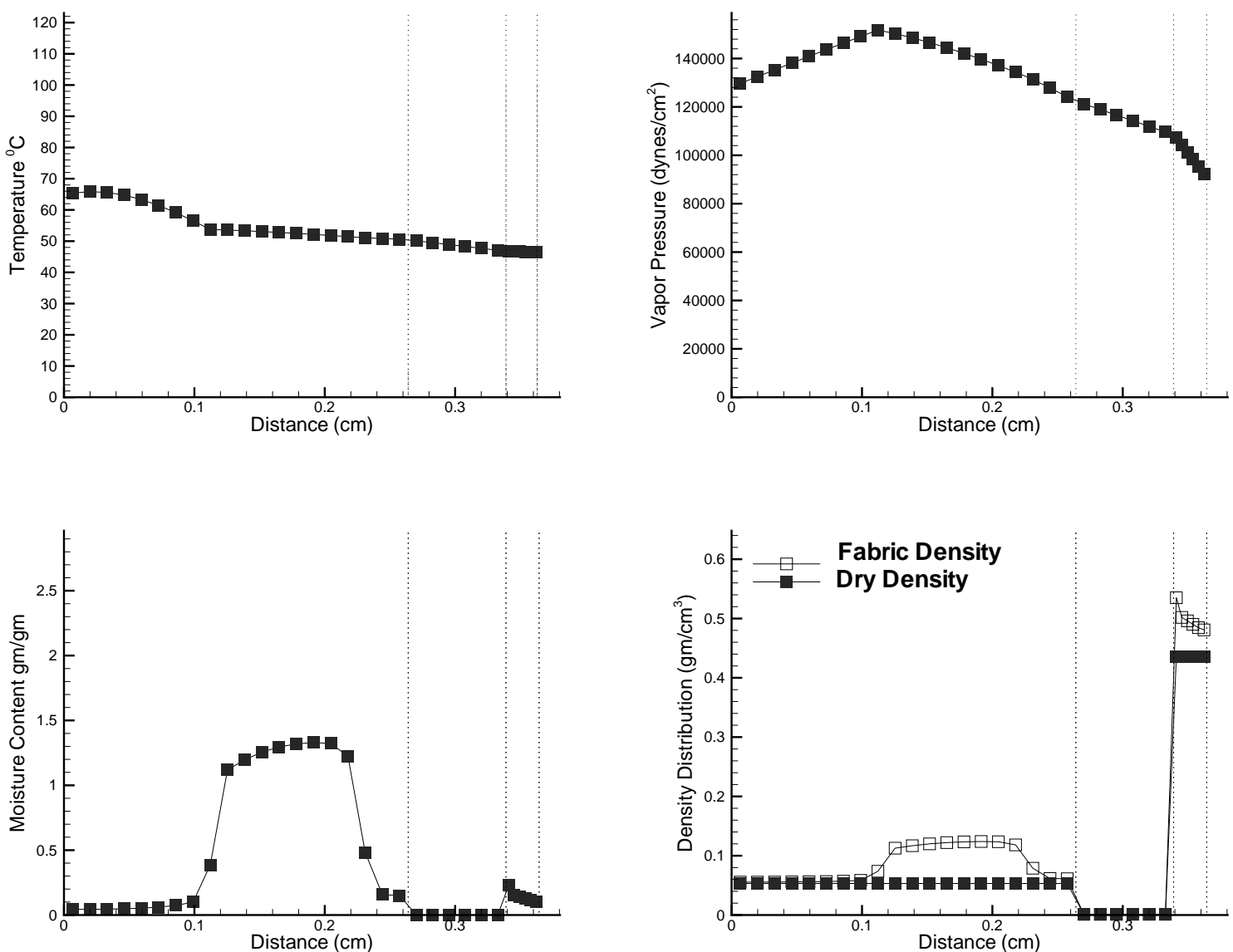

Figure 10: Simulation results at time $t=300 \mathrm{~s}$ for a wet thermal liner subjected to an external incident flux $2.5 \mathrm{~kW} / \mathrm{m}^{2}$. Temperature (top left sub-figure), vapor pressure (top right sub-figure), moisture content (bottom left sub-figure) and dry / wet density (bottom right sub-figure) has been shown as a function of distance measured from the front face of the thermal liner.

energy to the surroundings. The net flux, which is the difference between the incident flux and the reradiated amount is shown. For the back face, the radiative flux term is primarily because of heating of the back face, and as a result, it exhibits a pattern similar to the temperature profile at the back face (Figure 4).

Convective mass transfer at the outer boundaries is shown in Figure 16. A negative value of mass flux at the front face, indicates flux in the $-x$ direction and signifies removal of water vapor at the front face. A positive value of mass flux at the back face, indicates flux in the $+x$ direction and removal of vapor at the back face. At the front face, the mass flux initially increases rapidly with time as the water on the front face starts evaporating. It then stabilizes at an absolute value of $4.0 \times 10^{-5} \mathrm{~g} / \mathrm{cm}^{2} / \mathrm{s}$ until the front face is completely dry $(t=200 \mathrm{~s})$. Beyond $200 \mathrm{~s}$, the convective mass flux at the front face decreases and then stabilizes at 0.0 when the fabric is completely dry. After $t=750 \mathrm{~s}$, when the external radiation is cut off, the convective mass flux at the front face becomes positive, indicating absorption of moisture at the front face. At the back face, convective mass flux increases rapidly within $30-40 \mathrm{~s}$ and then stabilizes at a value of $8.0 \times 10^{-5} \mathrm{~g} / \mathrm{cm}^{2} / \mathrm{s}$. Note that the absolute value of mass flux at the back face is larger than that at the front face. As the face cloth dries up around $t=400 \mathrm{~s}$, there is a sharp jump in convective flux at the back face. Once the material is completely dry the flux settles down to zero until $t=750 \mathrm{~s}$. At this point the 


\section{Time $=\mathbf{4 0 0} .0$ seconds}
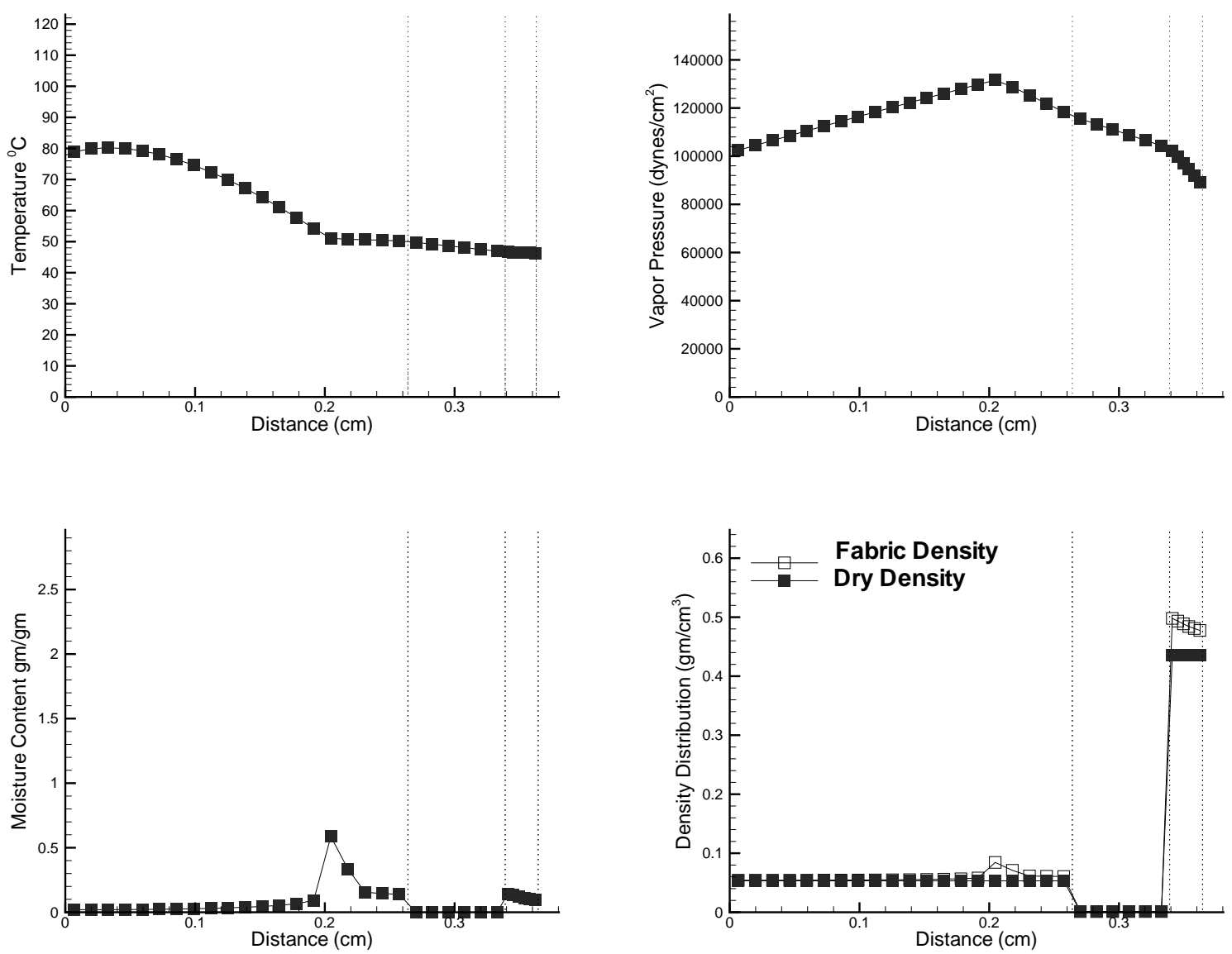

Figure 11: Simulation results at time $t=400 \mathrm{~s}$ for a wet thermal liner subjected to an external incident flux $2.5 \mathrm{~kW} / \mathrm{m}^{2}$. Temperature (top left sub-figure), vapor pressure (top right sub-figure), moisture content (bottom left sub-figure) and dry / wet density (bottom right sub-figure) has been shown as a function of distance measured from the front face of the thermal liner.

convective mass flux values at the back face becomes negative indicating absorption of moisture from the ambient atmosphere.

\subsection{Wet Turnout Coat Simulation}

A series of simulations were next conducted to study the performance of a typical turnout coat used by fire fighters. The turnout coat consists of three fabric layers, the shell (outermost layer, farthest from skin), the moisture barrier and the thermal liner. The shell material used in these calculations is Nomex III-Defender, the moisture barrier is Neo-Guard and the thermal liner is Aralite. Figure 17 shows the three fabric layers separated by an air gap of $1.0 \mathrm{~mm}$. Unlike the earlier calculations, the thermal liner was not separated into the batting and the face cloth, but instead treated as one material. Many fire fighter coats are made using these materials. To perform a simulation, the thickness, density, conductivity, specific heat and the optical properties of transmissivity and reflectivity were needed. These properties have been measured and tabulated by Lawson et al [23] and were directly used in our calculations.

For the turnout coat simulations, the left face of the outer shell is subjected to an external incident flux 


\section{Time $=\mathbf{5 0 0} .0$ seconds}
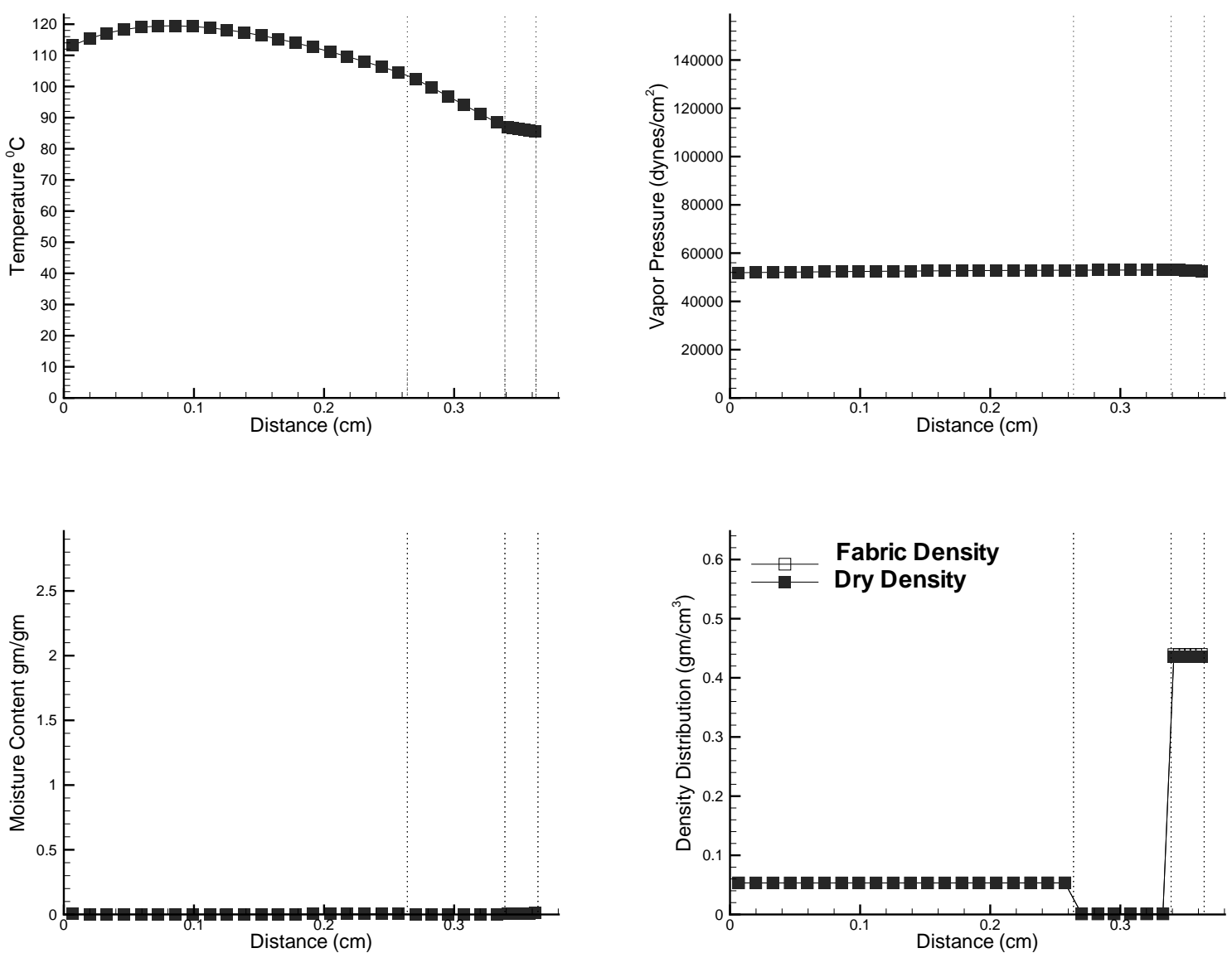

Figure 12: Simulation results at time $t=500 \mathrm{~s}$ for a wet thermal liner subjected to an external incident flux $2.5 \mathrm{~kW} / \mathrm{m}^{2}$. Temperature (top left sub-figure), vapor pressure (top right sub-figure), moisture content (bottom left sub-figure) and dry / wet density (bottom right sub-figure) has been shown as a function of distance measured from the front face of the thermal liner.

of $2.5 \mathrm{~kW} / \mathrm{m}^{2}$ starting at $t=0 \mathrm{~s}$ and lasting for approximately $750 \mathrm{~s}$. The simulation carries on until $850 \mathrm{~s}$ to allow for the fabrics to cool down. Wet turnout coat simulations were performed by assuming that only the thermal liner is initially wet and has a moisture content value of 1.0. Even though the outer shell and the moisture barrier are initially completely dry, water vapor can diffuse and convect to and condense on these fabric layers. Convective heat and mass transfer from the turnout coat occur at the front and back face, which are open to the atmosphere. It is assumed that the air-gap which is approximately $1 \mathrm{~mm}$ thick does not allow for a fully developed boundary layer. Therefore, the dominant mechanisms of heat and mass transfer at the fabric air-gap interface are through conduction and mass diffusion, respectively.

Figure 18 shows temperature time curves for the front and back faces of the turnout coat ensemble. The comparison in this case is for a thermal liner that is initially completely dry and a wet thermal liner. At the front face, the differences between dry turnout coat and wet turnout coat are not very significant. However, the back face shows large differences in temperature between dry and wet turnout coat ensemble. This is because for the wet turnout coat simulation, water is present only in the thermal liner, while the outer shell is completely dry initially. This moisture distribution affects the temperature at the back face much more significantly than that at the front face. The clothing ensemble clearly provides protection against the 


\section{Time $=\mathbf{8 0 0} .0$ seconds}
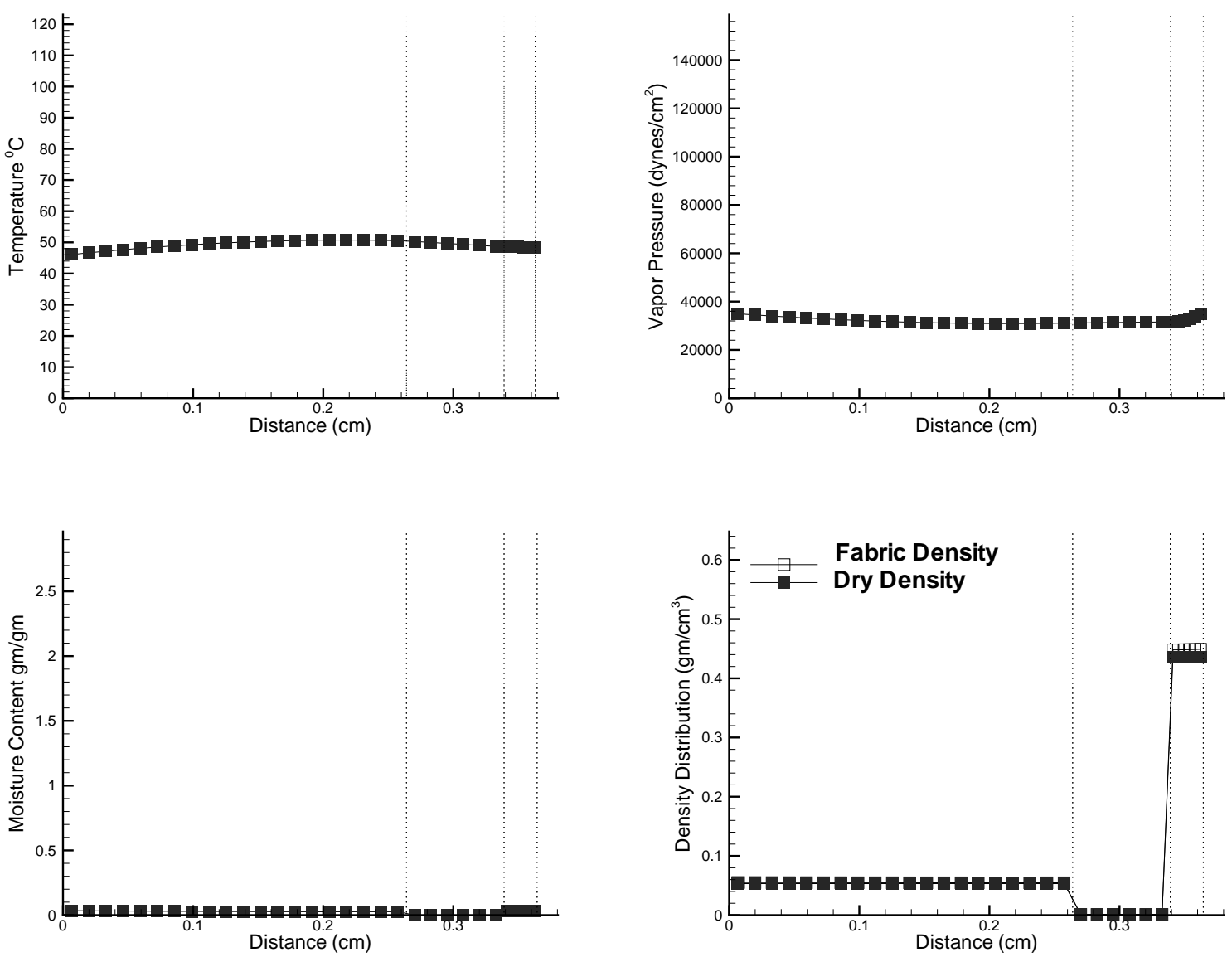

Figure 13: Simulation results at time $t=800 \mathrm{~s}$ for a wet thermal liner subjected to an external incident flux $2.5 \mathrm{~kW} / \mathrm{m}^{2}$. Temperature (top left sub-figure), vapor pressure (top right sub-figure), moisture content (bottom left sub-figure) and dry / wet density (bottom right sub-figure) has been shown as a function of distance measured from the front face of the thermal liner.

incident radiative flux. From the outside of the shell to the back of the thermal liner the temperature fell nearly $70^{\circ} \mathrm{C}$ for the wet thermal liner case, and $40^{\circ} \mathrm{C}$ for the dry thermal liner case.

Figures 19 and 20 show time dependent simulation results for a wet turnout coat ensemble, subjected to an external incident flux. These figures are similar to Figures 7-13 and the reader is directed to the discussion of Figure 7 for the significance of these plots. Dotted lines have been drawn on Figure 19 and 20 indicating the location of the interfaces. The various fabric layers have also been marked for clarity. Initially, at $t=0$ $s$, only the thermal liner is wet, with a moisture content value of 1.0. When the turnout coat is subjected to radiative heating, temperatures in the outer shell and moisture barrier increase significantly. At $t=100$ $s$ (Figure 19), the temperature in the thermal liner is relatively small and is almost constant through out its thickness. We observe that the front of the thermal liner has started drying. The vapor pressure curve peaks in this area, and shows a relatively lower value on either side. This indicates diffusion of water vapor from the thermal liner to the shell material and the moisture barrier. At $t=600 \mathrm{~s}$, (Figure 20) we observe higher temperatures in the thermal liner. The front part of the thermal liner is completely dry at this stage. We also observe convective mass loss at the back end of the liner. The wet fabric density is also shown to approach the dry density as moisture evaporates from the turnout coat ensemble. 


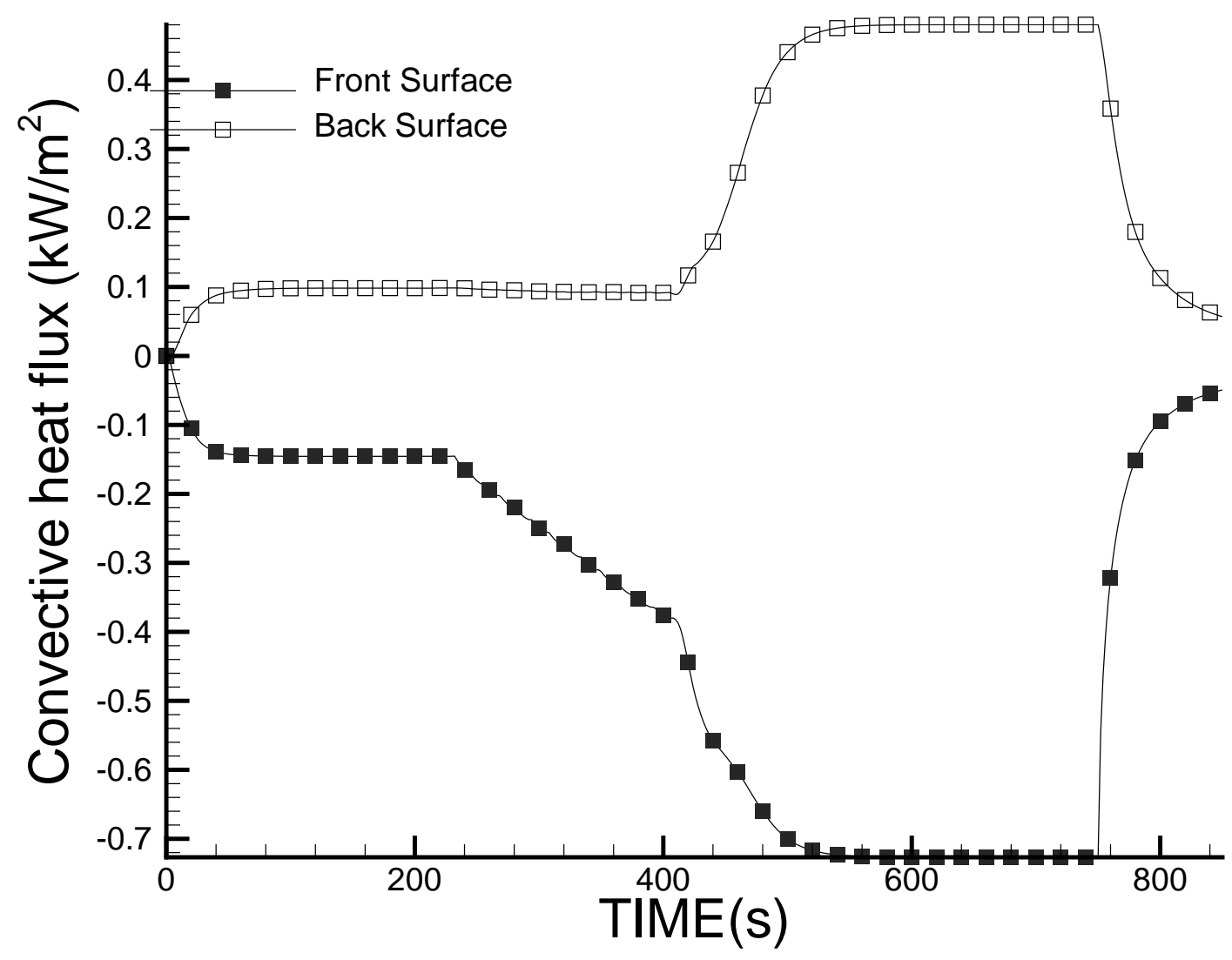

Figure 14: Numerical predictions of convective heat flux at the boundaries (front and back faces) as a function of time.

\section{Concluding Remarks}

Governing equations for transient heat and mass (moisture) transfer through fire fighter protective clothing when subjected to a radiative heat flux are derived from first principles. These equations account for the conductive, convective and radiative heat transfer through multi-layered fabric, as well as solve for convection, diffusion, evaporation, condensation, adsorption and desorption of moisture through the porous cloth. The effect of moisture on thermodynamic and transport properties is also accounted for in the governing equations. A simplified approximation for the governing equations are solved using an explicit, second order Runge-Kutta time stepping technique, subjected to appropriate boundary and interface conditions.

Numerical simulations are performed to obtain a detailed understanding of heat and mass transport through wet thermal liners and turnout coat ensemble. Results are compared with experimental data when available and are found to be in good agreement. Peak temperatures are predicted to within $10 \%$ accuracy. Moisture in the cloth evaporates when heated and part of it diffuses and recondenses on different parts of the fabric depending on local temperature. Moisture content and distribution along the thickness of the fabric is found to have a profound effect on the computed and measured temperature. We also conclude that moisture can have a significant effect on observed temperature and heat flux at the skin surface and can enhance or reduce burn injury (under different conditions) to the fire fighter. 


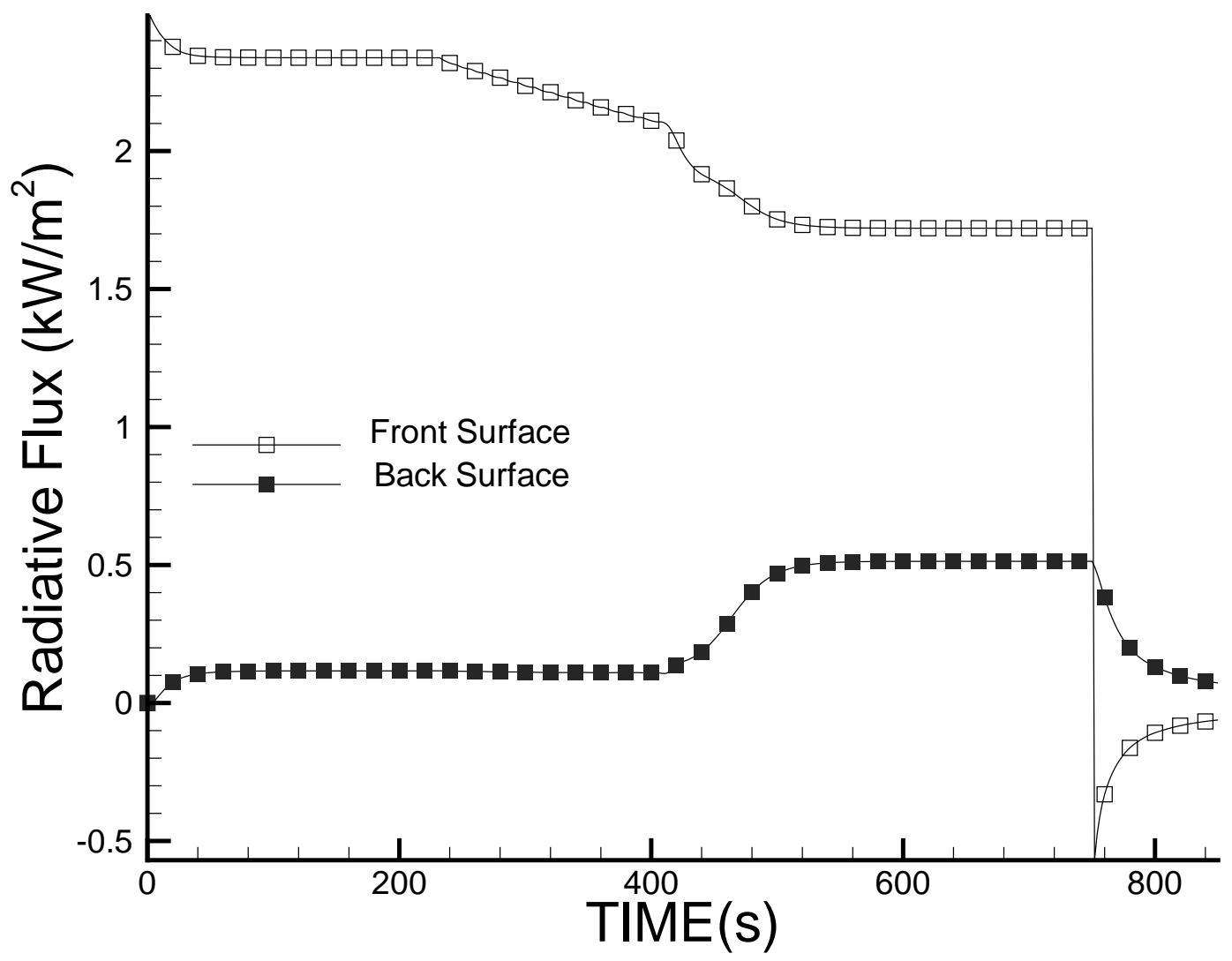

Figure 15: Numerical predictions of radiative heat flux at the boundaries (front and back faces) as a function of time.

\section{Directions for Future Research Activities}

Many more studies (similar to the one described in this report) need to be completed, to better understand the thermal performance of fire fighters' protective clothing, to design safe and effective protective garments, to predict burn injury and to develop tools for training and assisting the fire fighters subjected to different fire scenarios. The following is a list of research activities that we are planning for the next few years and which will be reported in subsequent publications.

1. A detailed sensitivity study on the effect of various parameters such as thermal conductivity, diffusion coefficient and heat capacity on the final result should be conducted. The influence of the convective heat and mass transfer correlations on the predicted results should be investigated further. Effect of geometric parameters such as thickness of the fabric layers and air gap on the final result should be studied carefully.

2. Effect of moisture on the optical properties of the fabric should be investigated. Note that in the current simulations, the optical properties of dry fabric layers have been used. Water has strong absorbtion bands in the near infrared regime, which should be included in the analysis. The sensitivity of optical properties on computed results should be studied carefully. Use of highly reflective and opaque fabrics 


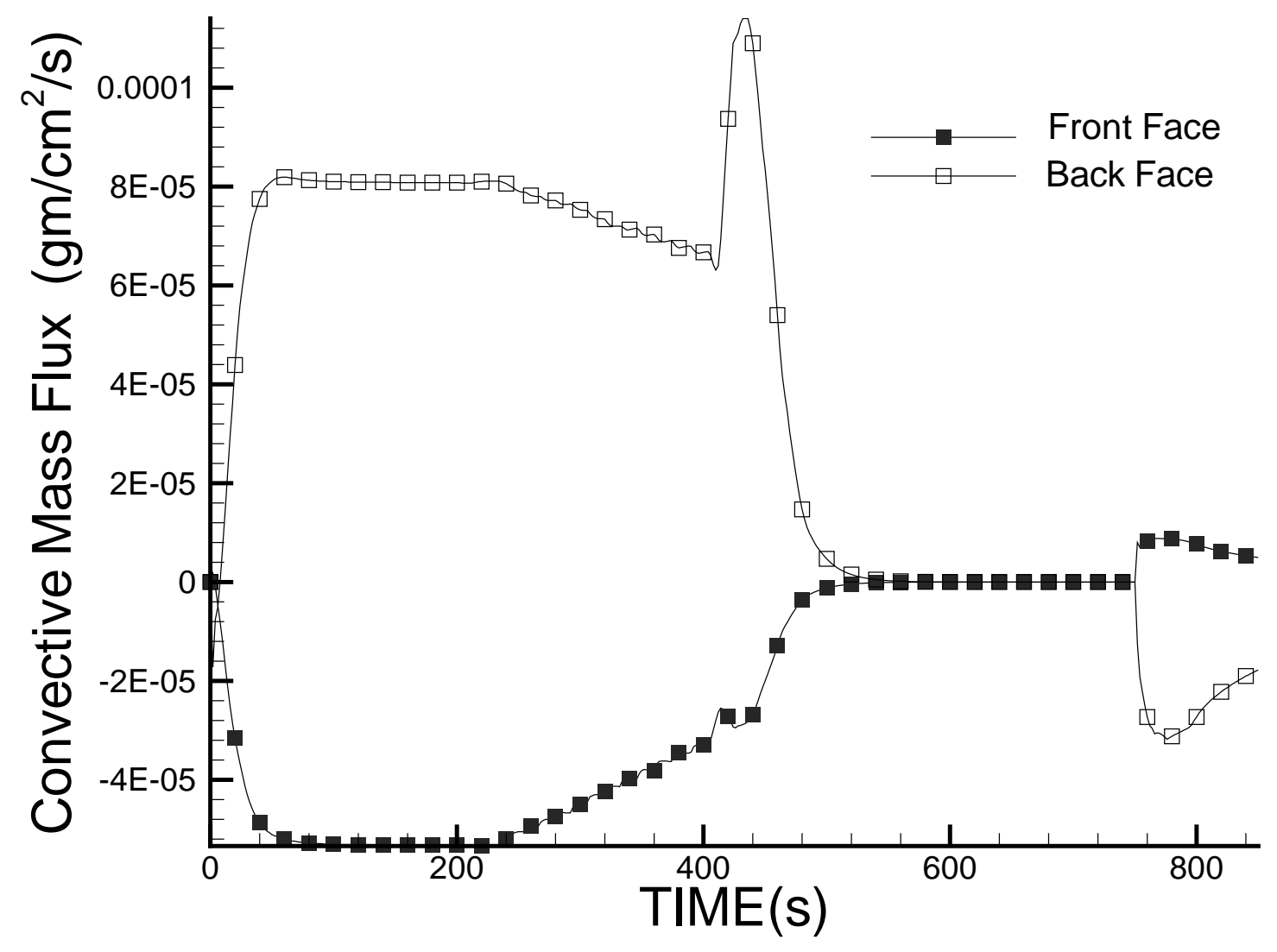

Figure 16: Numerical predictions of moisture mass flux at the boundaries (front and back faces) as a function of time.

in designing protective clothing can reduce fire fighter burn injury.

3. Adsorption isotherms measurements have been limited to three fabrics (Aralite, Neo-Guard and Nom ex III-Defender) that were directly relevant to this study. Measurement of adsorption isotherm for other fire fighter protective clothing should be completed, to make the model as general as possible. These measurements should cover a wide range of temperature.

4. More experiments with mass loss measurements, temperature measurements and heat flux measurements should be performed and compared with the numerical results to fully evaluate the model approximations.

5. A skin model should be coupled with the heat and mass transfer model to predict burn injury to the fire fighter. This model should account for energy removal by the flow of blood and sweat formation at the skin surface for which correlations should be developed [12]. Such a coupled model would be very useful in predicting time to onset of pain and $1^{\text {st }}$ and $2^{\text {nd }}$ degree burns from thermal radiation [24], [25], [26], [27].

6. Scald injuries and steam burns (when moisture condenses on the skin surface) should be investigated using model discussed in item 5. 


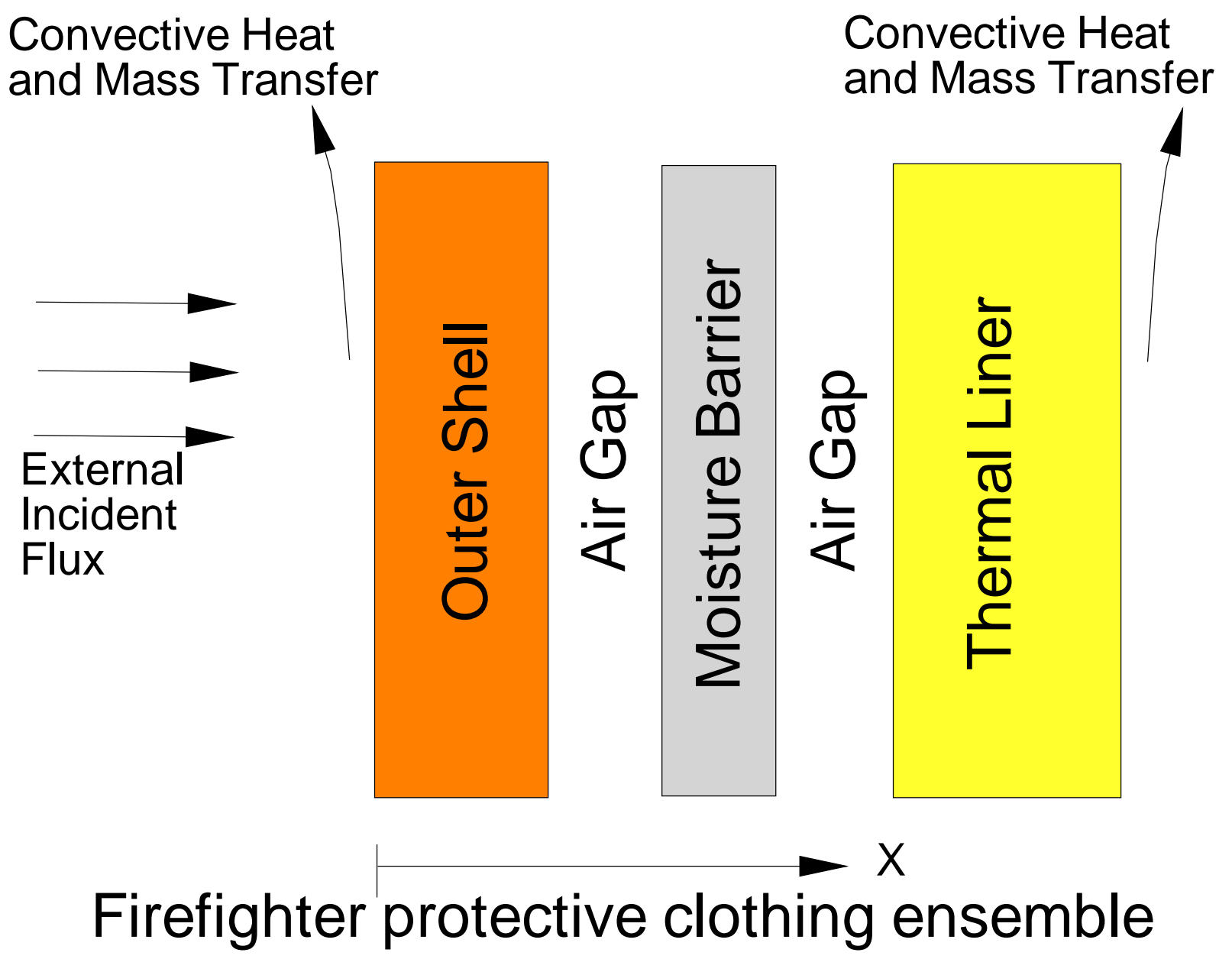

Figure 17: Schematic of a typical three layered turnout coat used as firefighter protective clothing, subjected to an incident radiative flux.

7. The heat and mass transfer model for porous clothing assemblies should be coupled with an engineering model of the human body to perform a detailed thermal analysis of human body clothing environment systems.

8. The thermal model of the human body - clothing system should obtain input data (heat flux, ambient temperature and humidity) from an FDS simulation [28] of a typical fire in an office building, town house, wildland fire or any other fire scenario in which a fire fighter will be present.

\section{Acknowledgments}

Appreciation is extended to Robert T. McCarthy of the United States Fire Administration for assistance provided during discussions related to fire fighter burn injuries and protective clothing performance. The authors would like to thank TAKASHI KASHIWAGI for his encouragement in studying the subject of transport processes in porous media and numerical modeling of condensed phases. Appreciation is extended to HOWARD BAUM for very helpful discussions on radiative heat transfer modeling. TINH NGUYEN and ERIC BYRD have assisted in obtaining the equilibrium sorption isotherms measurements for typical fire fighter protective clothing. We appreciate SIMON KAPLAN for providing measurements on thermo-optical proper- 


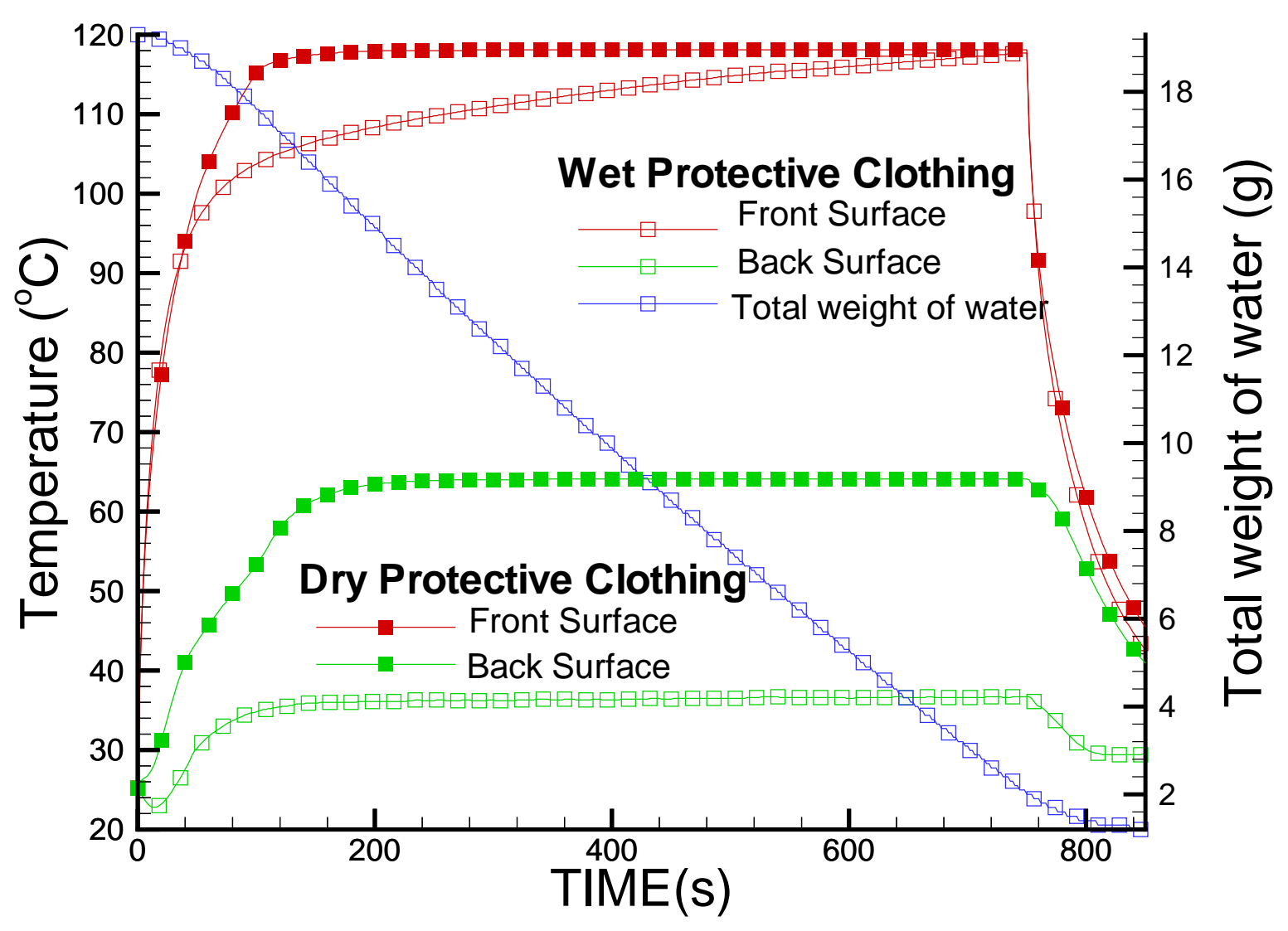

Figure 18: Comparison of numerically predicted temperature histories for the dry and wet turnout coat. For each case, numerical results have been shown for the front and back faces. The mass loss curve during evaporation of water from the turnout coat has also been shown on the $Y_{2}$ axis.

ties of the fabrics and for informative discussions. Finally, we thank ANTHONY HAMins, Dave Evans, NELSON BRYNER and DAN MADRYZKOWSKI for their overall support and guidance.

\section{References}

[1] J. R. Lawson. Fire fighter's protective clothing and thermal environments of structural fire fighting. Technical Report NISTIR 5804, National Institute of Standards and Technology, Gaithersburg, Maryland, October 1996.

[2] D. A. De Vries. Simultaneous transfer of heat and moisture in porous media. Transactions, American Geophysical Union, 39(5):909-916, 1958.

[3] J. R. Philip and D. A. DE Vries. Moisture movement in porous materials under temperature gradients. Transactions, American Geophysical Union, 38:222-232, 1957.

[4] E.R.G. Eckert and M. Faghri. A general analysis of moisture migration caused by temperature differences in an unsaturated porous medium. Int. J. Heat Mass Transfer, 23:1613-1623, 1980. 


\section{Time $=100.0$ seconds}
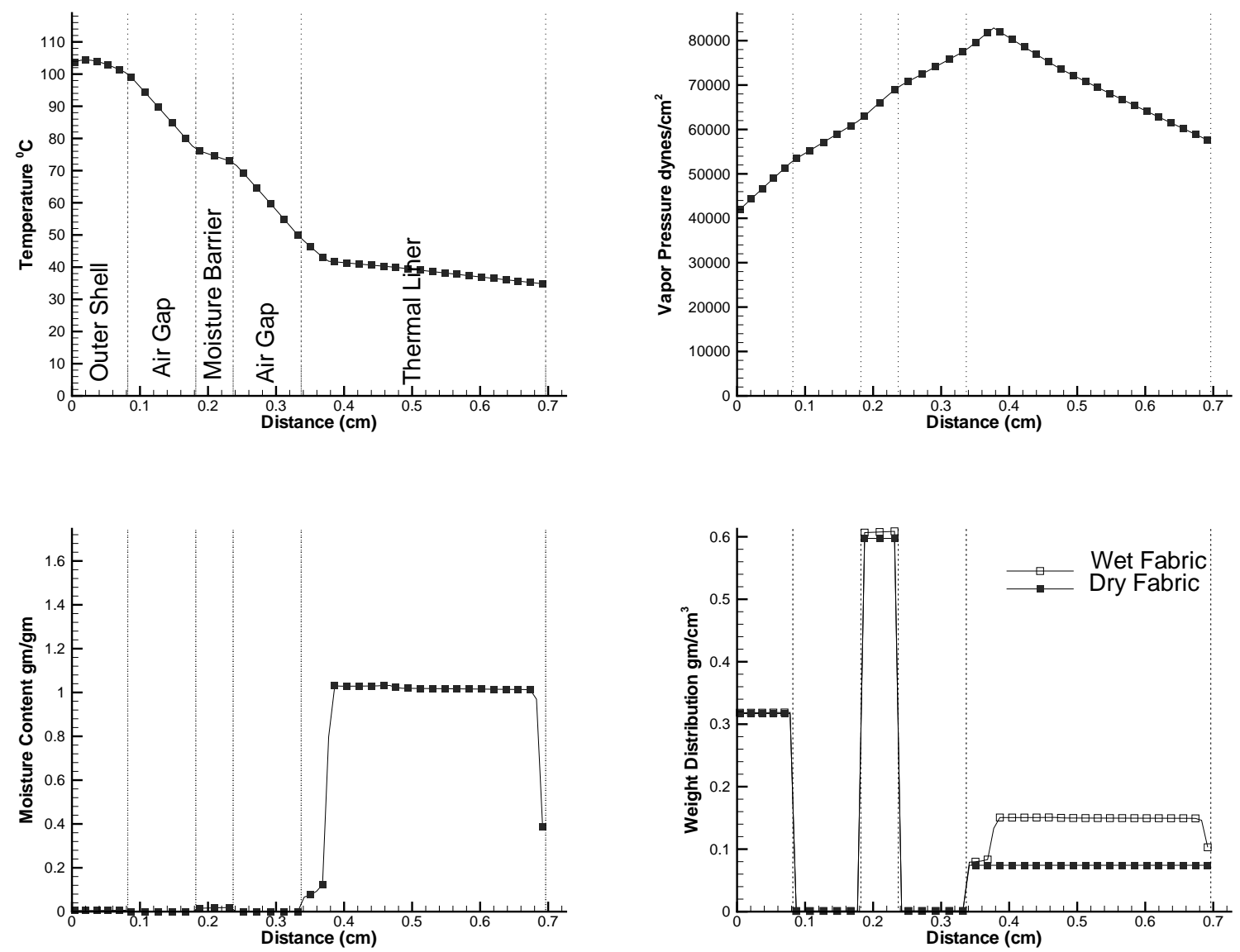

Figure 19: Simulation results at time $t=100 \mathrm{~s}$ for a wet turnout coat subjected to an external incident flux of $2.5 \mathrm{~kW} / \mathrm{m}^{2}$. Temperature (top left sub-figure), vapor pressure (top right sub-figure), moisture content (bottom left sub-figure) and dry / wet density (bottom right sub-figure) has been shown as a function of distance measured from the front face of the turnout coat.

[5] K. S. Udell. Heat transfer in porous media considering phase change and capillarity-the heat pipe effect. Int. J. Heat Mass Transfer, 28(2):485-495, 1985.

[6] K. S. Udell. Heat transfer in porous media heat from above with evaporation, condensation and capillary effects. J. Heat Transfer, 105:485-492, 1983.

[7] A. Bouddour, J.L. Auriault, M. Mhamdi-Alaoui, and J. F. Bloch. Heat and mass transfer in wet porous media in presence of evaporation - condensation. Int. J. Heat Mass Transfer, 41(15):2263-2277, 1998.

[8] P.W. Gibson. Review of numerical modeling of convection, diffusion, and phase change in textiles. In Computational Technologies for Fluid/Thermal/Structural/Chemical Systems with Industrial Applications- Volume II, volume 397, pages 125-139. ASME 1999, 1999.

[9] P. Nordon and H. G. David. Coupled diffusion of moisture and heat in hygroscopic textile materials. Int. J. Heat Mass Transfer, 10:853-866, 1967. 

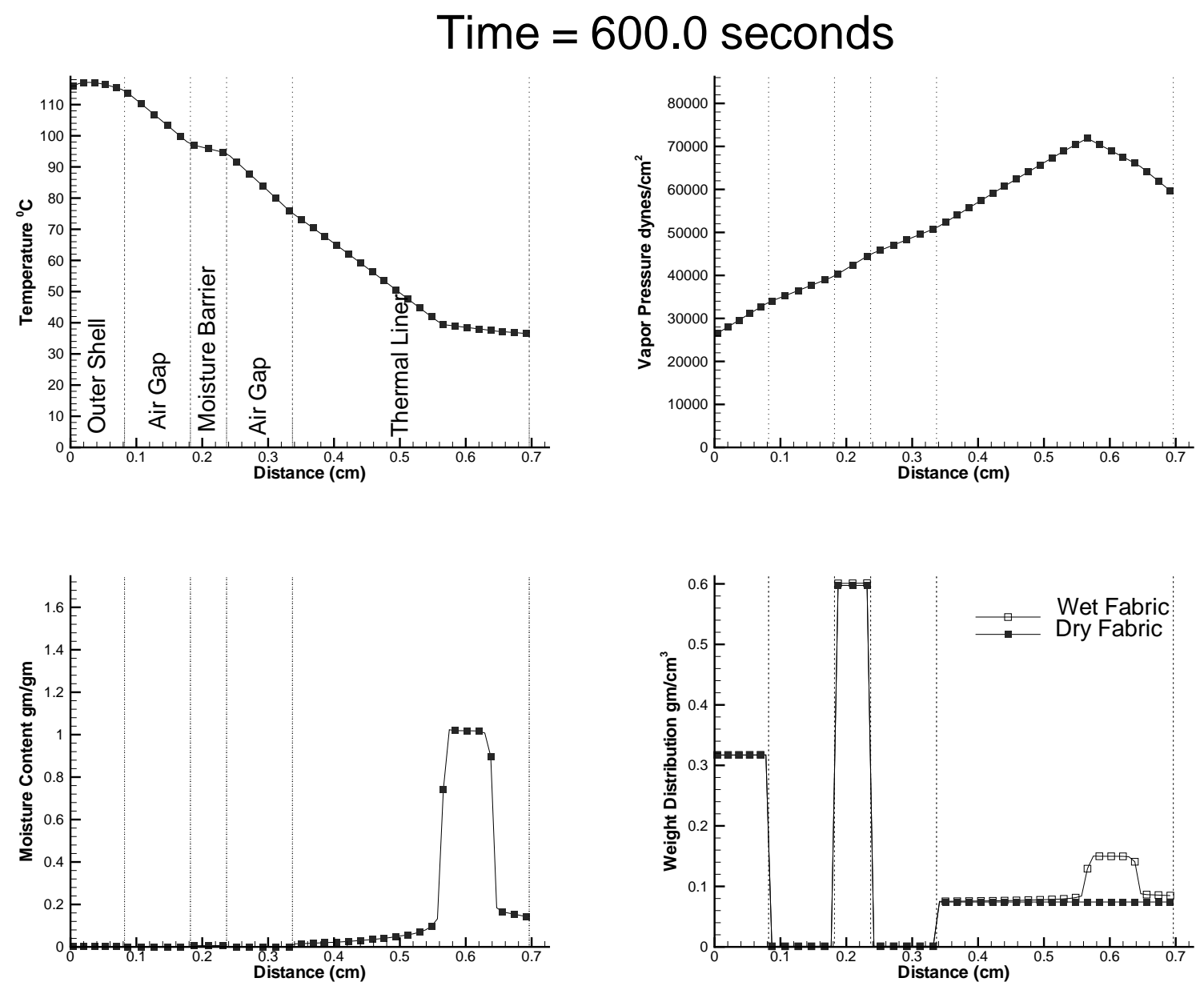

Figure 20: Simulation results at time $t=600 \mathrm{~s}$ for a wet turnout coat subjected to an external incident flux of $2.5 \mathrm{~kW} / \mathrm{m}^{2}$. Temperature (top left sub-figure), vapor pressure (top right sub-figure), moisture content (bottom left sub-figure) and dry / wet density (bottom right sub-figure) has been shown as a function of distance measured from the front face of the turnout coat.

[10] H. G. David and P. Nordon. Case studies of coupled heat and moisture diffusion in wool beds. Textile Research Journal, 39, February 1969.

[11] B. Farnworth. Mechanics of heat flow through clothing insulation. Textile Research Journal, 1983.

[12] G. J. Banford and W. Boydell. ICARUS: A Code for Evaluating Burn Injuries. Fire Technology, 31(4), December 1995.

[13] W.E. Mell and J. R Lawson. A heat transfer model for fire fighters' protective clothing. Technical Report NISTIR 6299, National Institute of Standards and Technology, Gaithersburg, Maryland, January 1999.

[14] A. M. Schneider and B. N. Hoschke. Heat transfer through moist fabrics. Textile Res. J., 62(2):61-66, 1992.

[15] J. Baer. Dynamics of Fluids in Porous Media. American Elsevier Publishing Company, Inc., New York, NY, 1973. 
[16] J. Crank and G. S. Park. Diffusion in Polymers. Springer-Verlag Inc., New York, NY, 1968.

[17] I. C. Watt and R. L. DÁrcy. Water-vapor adsorption isotherms of wool. J. Text. Inst., 7:298-307, 1979.

[18] A. R. Urquhart and A. M. Williams. The moisture relations of cotton: The effect of temperature on the absorption of water by soda-boiled cotton. J. Text. Inst., 15:559-572, 1924.

[19] J. Quintiere. Radiative Characteristics of Fire Fighter's Coat Fabrics. Fire Technology, 10(2), May 1974.

[20] M. N. Ozisik. Heat Transfer A Basic Approach. McGraw-Hill, Inc., New York, NY, 1985.

[21] J. P. Holman. Heat Transfer. McGraw-Hill, Inc., New York, NY, 1990.

[22] H. S. Carslaw and J. C. Jaeger. Conduction of Heat in Solids. Oxford University Press, New York, NY, 1947.

[23] J. R Lawson and W. D. Walton. Estimates of thermal properties for fire fighters' protective clothing materials. Technical Report to be published, National Institute of Standards and Technology, Gaithersburg, Maryland, 2002.

[24] A. Stoll and L. C. Greene. Relationship between pain and tissue damage due to thermal radiation. Journal of Applied Physiology, 14:373-383, 1959.

[25] F. C. Henriques. Study of thermal injury v. the predictability and the significance of thermally induced rate processes leading to irreversible epidermal injury. Archives of Pathology, 43:489-502, 1947.

[26] A. Stoll. The Role of Skin in Heat Transfer, volume 4 of Advances in Heat Transfer, pages 115-141. Academic Press, New York, NY, 1967.

[27] A. Stoll and M. A. Chianta. Heat transfer through fabrics as related to thermal injury. Transactions New York Academy of Sciences, 33(7):649-670, 1971.

[28] K.B. McGrattan, H.R. Baum, R.G. Rehm, A. Hamins, G.P. Forney, J. A. Floyd, and S. Hostikka. Fire Dynamics Simulator (Version 2) - Technical Reference Guide. Technical Report NISTIR 6783, National Institute of Standards and Technology, Gaithersburg, Maryland, November 2001. 